NBER WORKING PAPER SERIES

\title{
REPUTATION CYCLES AND EARNINGS DYNAMICS
}

\author{
Boyan Jovanovic \\ Julien Prat \\ Working Paper 25252 \\ http://www.nber.org/papers/w25252 \\ NATIONAL BUREAU OF ECONOMIC RESEARCH \\ 1050 Massachusetts Avenue \\ Cambridge, MA 02138 \\ November 2018
}

We are grateful to Sai Ma and Hargungheet Singh for excellent research assistance and to Jess Benhabib, Emmanuel Farhi, Jean-Michel Grandmont, Fatih Guvenen and Dimitris Papanikolaou for their insightful suggestions. We thank participants of the SED 2015, Barcelona GSE summer forum 2015, NASM 2016, and Minnesota Macro 2016 for their comments. Julien Prat acknowledges the support of the Investissements d'Avenir grant (ANR-11-IDEX-0003/Labex Ecodec/ANR-11-LABX-0047). The views expressed herein are those of the authors and do not necessarily reflect the views of the National Bureau of Economic Research.

NBER working papers are circulated for discussion and comment purposes. They have not been peer-reviewed or been subject to the review by the NBER Board of Directors that accompanies official NBER publications.

(C) 2018 by Boyan Jovanovic and Julien Prat. All rights reserved. Short sections of text, not to exceed two paragraphs, may be quoted without explicit permission provided that full credit, including (c) notice, is given to the source. 
Reputation Cycles and Earnings Dynamics

Boyan Jovanovic and Julien Prat

NBER Working Paper No. 25252

November 2018

JEL No. D31

\section{ABSTRACT}

Cyclical patterns in earnings can arise when contracts between firms and their workers are incomplete, and when workers cannot borrow or lend so as to smooth their consumption. Earnings cycles generate occasional large changes in earnings, consistent with some recent empirical findings. At the calibrated parameter values, financial constraints promote investment in reputation - an intangible capital form - in contrast to their documented inhibiting effect on investment in tangible capital.

Boyan Jovanovic

New York University

Department of Economics

19 W. 4th Street, 6th Floor

New York, NY 10012

and NBER

Boyan.Jovanovic@nyu.edu

Julien Prat

CREST

15 Boulevard Gabriel Peri

92245 Malakoff Cedex

France

julien.prat@ensae.fr 


\title{
Reputation Cycles and Earnings Dynamics
}

\author{
Boyan Jovanovic* and Julien Prat ${ }^{\dagger}$
}

October 2018

\begin{abstract}
Cyclical patterns in earnings can arise when contracts between firms and their workers are incomplete, and when workers cannot borrow or lend so as to smooth their consumption. Earnings cycles generate occasional large changes in earnings, consistent with some recent empirical findings. At the calibrated parameter values, financial constraints promote investment in reputation - an intangible capital form - in contrast to their documented inhibiting effect on investment in tangible capital.
\end{abstract}

Keywords: Reputation, Career Concerns, Earnings Dynamics, Endogenous Fluctuations.

\section{Introduction}

Recent analyses of large panel data sets reveal that earnings fluctuate in ways that cannot be captured by the linear models commonly used in the literature on earnings dynamics. In particular, earnings data show occasional changes far larger than predicted by a log-normal distribution - see Guvenen, Karahan, Ozkan and Song (2015) and Arellano, Blundell and Bonhomme (2017), henceforth GKOS and ABB respectively.

*Economics Department, NYU. bj2@nyu.edu

${ }^{\dagger}$ CNRS, CREST, Ecole Polytechnique. julien.prat@ensae.fr. We are grateful to Sai Ma and Hargungheet Singh for excellent research assistance and to Jess Benhabib, Emmanuel Farhi, Jean-Michel Grandmont, Fatih Guvenen and Dimitris Papanikolaou for their insightful suggestions. We thank participants of the SED 2015, Barcelona GSE summer forum 2015, NASM 2016, and Minnesota Macro 2016 for their comments. Julien Prat acknowledges the support of the Investissements d'Avenir grant (ANR-11-IDEX-0003/Labex Ecodec/ANR-11-LABX-0047). 
These unusual events are routinely attributed to external shocks such as layoffs triggering falls off the job ladder. Since large income fluctuations reduce the welfare of risk-averse agents, it seems natural to conjecture that they are not initiated by them. We show that career-concerned agents may in fact choose to go through periods of low and high effort. We call these oscillations reputation cycles.

We change the career concerns model of Holmström (1999) by assuming that the agent is risk-averse and cannot borrow or lend. Since reputational investment pays off only in the future, a worker's incentive to maintain or improve her reputation depends on her discount factor. If she is risk averse, her discount factor depends negatively on her consumption growth. When the worker cannot smooth her consumption by other means, ${ }^{1}$ this force can then give rise to cycles in her effort, and thus in her earnings. The mechanism works as follows: When effort is low, the worker's consumption is low relative to her future consumption which means that her discount factor is also low. This reduces her incentive to create a good reputation and low effort is self fulfilling. Conversely, in a period of high effort, current consumption is higher than future consumption, which means that the discount factor is also large and so high effort is self fulfilling too. In other words, the discount factor and reputational concerns are high because consumption growth is low.

The model delivers several insights. First, evidence has shown that tangible investment is inhibited by liquidity constraints - Fazzari, Hubbard and Petersen (1988). We find the opposite at least as far as reputational investment - an intangible - is concerned. Under the set of calibrated parameters, cyclical paths may entail higher average reputation capital than does the rest point solution of the model, although cyclical paths exist only if the agent cannot borrow or lend.

Second, in terms of pure theory, we show the existence of a new class of equilibria. Repeated games are known to have equilibria that shift from period to period with no change in the payoff functions in the stage game - e.g., Green and Porter (1984), Mailath and Samuelson (2016, Sec. 4.3.1). In dynamic games with type uncertainty of which ours is an example, Markovian equilibria also can entail fluctuating effort e.g., Board and Meyer-ter-Vehn (2013) and Cisternas (2018). With a risk averse agent, however, we show that the Holmström model acquires a Markovian, cyclical equilibrium: At each belief about the agent's quality, there are three Markov-equilibrium actions. Moreover, cyclical equilibria can arise even though the agent has a publicly

\footnotetext{
${ }^{1}$ Kaplan, Weidner and Violante (2014) estimate that one third of U.S. consumers are hand to mouth.
} 
known retirement date.

Third, we explore the model's quantitative properties using micro-data on earnings dynamics to calibrate its parameters. The variance coefficients can be recovered from Lange's (2007) estimates about the speed of employer learning. In the model, income growth decomposes into small, fully persistent changes and larger, meanreverting changes roughly as U.S. panel data on earnings show: GKOS and ABB detect significant deviations from lognormality in earnings data, with a small but noticeable share of individuals experiencing very large changes. They also find that the degree of persistence appears to be non-linear in the size of the shocks as large shocks exhibit much stronger mean reversion than small shocks. Hubmer (2018) shows that a life-cycle version of the standard job ladder model can capture the large negative skewness and high excess kurtosis of earnings data. Our analysis indicates that endogenous fluctuations in effort can also help to explain these large deviations from the log-normal framework, while addressing the systematic correlation between the persistence and the size of income shocks.

Finally, the model generates a negative relation between exogenous income noise and the volatility of earnings: Income can become more volatile when reputational concerns rise in response to a reduction in the noise accompanying the relation between effort and output. Agency theory suggests that a reduction in noise raises the incentives to provide effort, and we show that this can also help explain the rise in the volatility of earnings. For cycles in effort to exist, incentives to provide effort cannot be too weak. Noise with which the agent's output is observed deters effort and it also obstructs the formation of cycles. This bears on the question of why income inequality has risen - Song, Price, Guvenen, Bloom, von Wachter (2018), henceforth SPGBW. Paradoxically, we find that an increase in inequality could have in part been caused by a decline in exogenous volatility. In particular, a decline in the noisiness of technology will strengthen reputational concerns and may raise equilibrium income volatility. Hence what SPGBW measure as a rise in the volatility of observed income may in fact be the result of a decline in exogenous output noise.

The plan of the paper is as follows. Section 2 lays out the model. Section 3 deal$\mathrm{s}$ with reputation cycles when disutility is linear in effort, where the mechanism is more transparent, while Section 4 deals with convex disutility functions. We explain in Section 5 how the model can be parametrized, and explore its quantitative implications. Then we review major hypotheses for endogenous cycles in Section 6.1 so as to highlight the novelty of reputation cycles. Several extensions are discussed in 
Section 6.2, while Section 7 concludes. All the proofs are relegated to the Appendix.

\section{Model}

In Holmström (1999) a risk-neutral agent faces a spot market with risk-neutral buyers. The unique equilibrium then entails a monotonic time path of effort. We will show that if one assumes that the agent is risk averse, multiple equilibria arise and in some of them effort follows a 2-period cycle. We assume the following:

A1. Non-contractible effort and output;

A2. Risk averse agent facing short-term risk-neutral buyers;

A3. Agent cannot borrow or lend.

We maintain these assumptions throughout.

Production.-Each period an agent produces output $y_{t}$ by exerting hidden effort $a_{t}$ :

$$
y_{t}=\theta_{t}+a_{t}+\varepsilon_{t} .
$$

Here $\varepsilon_{t} \sim N\left(0, \sigma_{\varepsilon}^{2}\right)$ is an i.i.d. shock. The variable $\theta$ is the agent's efficiency; $\theta$ is unknown, even to the agent, and the common prior is $\mathcal{N}\left(\theta_{0}, \sigma_{\theta}^{2}\right)$.

Earnings.-Contracts in which the period- $t$ payment is contingent on $y_{t}$ are not feasible. Instead, buyers pay the agent up front and the payment reflects the market belief at the beginning of the period. Thus the agent exerts effort only so as to raise her future income. Output has a persistent effect on prices because it affects the market's belief about the agent's efficiency which fluctuates over time as follows

$$
\theta_{t}=\theta_{t-1}+\nu_{t}, \text { with } \nu \sim \mathcal{N}\left(0, \sigma_{\nu}^{2}\right)
$$

Information.-Each period the agent chooses an $a$ from a compact and convex set $\mathcal{A} \subseteq \mathbb{R}_{+}$while everyone observes $y$. The agent can also observe $\theta+\varepsilon$ but not its components. The market's participants observe $y$ only, but since they will deduce $a$ from equilibrium, they too will be able to infer $\theta+\varepsilon$. However, the market and the agent observe different signals whenever the agent deviates and take a different action than the one anticipated by the market. We account for such divergence by using $E^{*}[\cdot]$ and $E[\cdot]$ to denote the market's and the agent's expectation, respectively. 
Timing.-Within a period, events unfold as follows:

1. There are many identical and risk neutral potential buyers, and they get zero rents. Hence the up-front payment the agent gets is $E^{*}\left[y_{t} \mid y^{t}\right]$, where $y^{t} \equiv$ $\left\{y_{s}\right\}_{s=0}^{t-1}$ is her public history.

2. The agent chooses $a_{t}$, privately.

3. Output $y_{t}$ is realized, and it becomes part of the publicly observed history $y^{t+1}$.

Preferences. - The agent is risk averse and infinitely lived. Her preferences as of date 0 read

$$
\sum_{t=0}^{\infty} \beta^{t} \mathbf{U}\left(c_{t}, a_{t}\right)
$$

with $\beta \in(0,1)$ denoting the discount factor.

Equilibrium.-We will examine equilibria in pure strategies in which the agent's behavior along the path of play is a function of time only. An equilibrium must satisfy the following three requirements. First, payments are set competitively by the market. Second, the agent chooses her effort so as to maximize expected payoffs. Third, the market conjecture $a_{t}^{*}$ is verified.

Definition 1 A deterministic equilibrium is a profile $\left\{a_{t}, a_{t}^{*}, c_{t}\right\}_{t=0}^{\infty}$ such that $\left\{a_{t}\right\}_{t=0}^{\infty}$ depends on time only and:

1. Expected profits are zero in every period as $c_{t}=E^{*}\left[y_{t} \mid y^{t}\right]$.

2. Effort $a_{t}$ is chosen optimally

$$
a_{t}=\arg \max _{\hat{a}_{t} \in \mathcal{A}} E\left[\sum_{s=0}^{\infty} \beta^{s} \mathbf{U}\left(c_{s}, \hat{a}_{s}\right)\right], \text { for all } t=0,1, \ldots
$$

3. The market's conjecture $\left\{a_{t}^{*}\right\}_{t=0}^{\infty}$ is correct, so that $a_{t}^{*}=a_{t}$ for all $t=0,1, \ldots$

Market beliefs. - In order to interpret the output signal, the market's participants need to conjecture the agent's behavior. For a given conjecture $\left\{a_{s}^{*}\right\}_{s=0}^{t-1}$, a sufficient statistic for the information revealed about $\theta_{t}$ is the sequence $x^{t}=\left\{x_{s}\right\}_{s=0}^{t-1}$, where

$$
x_{t} \equiv y_{t}-a_{t}^{*}=\theta_{t}+a_{t}-a_{t}^{*}+\varepsilon_{t} .
$$


The market treats $x$ as the signal. On path, i.e., when $a_{t}=a_{t}^{*}, x_{t} \sim N\left(\theta_{t}, \sigma_{\varepsilon}^{2}\right)$. Because $\nu$ and $\varepsilon$ are normal, the posterior is also normal: $\theta_{t} \sim \mathcal{N}\left(m_{t}, \sigma_{\theta, t}^{2}\right)$. The posterior variance evolves deterministically as

$$
\sigma_{\theta, t}^{2}=\frac{1}{\sigma_{\theta, t-1}^{-2}+\sigma_{\varepsilon}^{-2}}+\sigma_{\nu}^{2}
$$

and converges in the long-run to $\bar{\sigma}_{\theta}^{2}$, whose value is obtained setting $\sigma_{\theta, t}=\sigma_{\theta, t-1}$ in (5) so that ${ }^{2}$

$$
\bar{\sigma}_{\theta}^{-2}=\frac{\sigma_{\varepsilon}^{-2}}{2}\left(\sqrt{1+4 \frac{\sigma_{\varepsilon}^{2}}{\sigma_{\nu}^{2}}}-1\right) .
$$

We will focus on stationary learning (Holmström, 1999, Sec. 2.2). Hence we assume that the agent's initial $\theta$ is drawn from $\mathcal{N}\left(\theta_{0}, \bar{\sigma}_{\theta}^{2}\right)$. Then $\sigma_{\theta, t}=\bar{\sigma}_{\theta}$ remain constant and time is not a state. The market beliefs' posterior mean $m$ obeys the following law of motion

$$
m_{t+1}=E^{*}\left[\theta_{t+1} \mid m_{t}, x_{t}\right]=\lambda m_{t}+(1-\lambda) x_{t}, \text { where } \lambda \equiv \frac{\sigma_{\varepsilon}^{2}}{\sigma_{\varepsilon}^{2}+\bar{\sigma}_{\theta}^{2}} .
$$

As (4) shows, by deviating from $a_{t}^{*}$, the agent can raise $x_{t}$, manipulate $m_{t+1}$ and drive a wedge between her own belief and that of the market. We now analyze the agent's incentives to do that.

\subsection{Investment in reputation}

The zero-profit condition for market's participants is satisfied when $c_{t}=E^{*}\left[y_{t} \mid y^{t}\right]=$ $m_{t}+a_{t}^{*}$, with $m_{t}$ obeying the law of motion (7). We focus on equilibria in which $a_{t}^{*}$ is deterministic. The agent's FOC must hold at $a^{*}$ :

$$
a_{t}^{*}=\arg \max _{a_{t} \in \mathcal{A}} E\left[\sum_{s=0}^{\infty} \beta^{s} \mathbf{U}\left(m_{s}+a_{s}^{*}, a_{s}\right)\right], \text { for all } t=0,1, \ldots
$$

This requirement ensures that: (i) given the law of motion of beliefs derived in the previous subsection, equilibrium strategies satisfying (8) maximize the agent's utility; (ii) given the equilibrium actions, beliefs are updated via Bayes rule which is well

\footnotetext{
${ }^{2}$ Observe that $\partial \bar{\sigma}_{\theta}^{-2} / \partial \sigma_{\varepsilon}<0$ and $\partial \bar{\sigma}_{\theta}^{-2} / \partial \sigma_{\nu}<0$ as fluctuations in output and efficiency lower stationary precision.
} 
defined for all $\hat{a}$ since all output levels occur with positive probability at $a^{*}$. Solutions to problem (8) are therefore optimal.

We now establish a sufficient condition for the existence of deterministic equilibria. Whenever the agent deviates, she drives a persistent wedge between her belief and that of the market. Thus a deviation that is not attractive on the equilibrium path might nonetheless be profitable off path, and so the necessary condition is not generally sufficient. We now show that this concern does not apply when $U(\cdot)$ is in the CARA class.

Proposition 1 Assume that the per-period utility function is CARA:

$$
\mathbf{U}(c, a)=U(c-g(a))=-\exp (-\gamma(c-g(a))), \text { with } \gamma>0 .
$$

A deterministic profile $\left\{a_{t}^{*}\right\}_{t=0}^{\infty}$ is an equilibrium profile if and only if

$$
\mathbf{U}_{2}\left(c_{t}, a_{t}^{*}\right)=\frac{1-\lambda}{\lambda} \sum_{s=t+1}^{\infty}(\beta \lambda)^{s-t} E_{t}^{*}\left[\mathbf{U}_{1}\left(c_{s}, a_{s}^{*}\right)\right] .
$$

The proof is in the Appendix and it proceeds in two steps. First, we establish that optimal strategies are deterministic on and off the equilibrium path whenever the market's conjecture $a_{t}^{*}$ is deterministic. This result holds because shocks to the agent's reputation are permanent. Then income shocks, which reflect the history $y^{t}$, will in turn be permanent because buyers are risk neutral and because their beliefs have the martingale property. But permanent income shocks matter for the decisions of the agent only if her preferences display a wealth effect, which can be turned off using the CARA specification. We can therefore focus, without loss of generality, on sequences of deterministic actions. This restriction enables us to prove the concavity of the agent's objective in the space of action sequences, thus directly establishing the sufficiency of the first order condition. ${ }^{3}$

The left-hand side of (10) is the marginal disutility, while the right-hand side is the discounted benefit because a marginal increase in effort raises the posterior mean at date $s$ by $\partial m_{s} / \partial a_{t}=(1-\lambda) \lambda^{s-t-1}$, for all $s>t .{ }^{4}$ Since $c$ is increasing in $m$ and $m$ is a martingale, expected consumption depends on the agent's current reputation.

\footnotetext{
${ }^{3} \mathrm{~A}$ recent paper by Cisternas (2016) provides bounds guaranteeing sufficiency in more general settings in which optimal actions may depend on the posterior $m$.

${ }^{4}$ Eq. (10) is the counterpart of Holmström's (1999), eq. (22), with the essential addition of the terms $\mathbf{U}_{2}\left(c_{t}, a_{t}^{*}\right)$ and $E_{t}^{*}\left[\mathbf{U}_{1}\left(c_{s}, a_{s}^{*}\right)\right]$.
} 
Hence the optimality condition (10) varies with $m_{t}$ and in general so would $a_{t}^{*}$, contrary to our assumption that $a_{t}^{*}$ depends on $t$ only. To ensure that $E_{t}\left[\mathbf{U}_{1}\left(c_{s}, a_{s}^{*}\right) / \mathbf{U}_{2}\left(c_{t}, a_{t}^{*}\right)\right]$ is not affected by $m_{t}$, we neutralize the wealth effect by assuming that the agent's utility function is CARA. This restriction ensures that the agent's discount factor no longer depends on $m_{t}$ because it is the forecastable component of $m_{s}$ for all $s>t$.

Proposition 2 When the per-period utility function is CARA, a deterministic profile $\left\{a_{t}^{*}\right\}_{t=0}^{\infty}$ is an equilibrium profile if and only if

$$
g^{\prime}\left(a_{t}^{*}\right)=\frac{1-\lambda}{\lambda} \sum_{s=t+1}^{\infty}(r \lambda)^{s-t} \exp \left(\gamma\left[a_{t}^{*}-g\left(a_{t}^{*}\right)-\left(a_{s}^{*}-g\left(a_{s}^{*}\right)\right)\right]\right)
$$

where

$$
r \equiv \beta \exp \left(\frac{\gamma^{2} \sigma_{\nu}^{2}}{2}\right)
$$

The effective discount factor $r$ is higher than $\beta$ because consumption is stochastic and the agent is prudent $\left(U^{\prime \prime \prime}(\cdot)>0\right)$. A mean-preserving spread in consumption raises expected marginal utility in future periods, and so makes the agent more patient. This precautionary motive is stronger when the coefficient of risk-aversion $\gamma$ is large because $\gamma$ governs the curvature of the marginal utility function. The discount factor $r$ is also increasing in $\sigma_{\nu}^{2}$ because beliefs, and thus consumption, are more volatile when types are less persistent. By contrast, $r$ does not depend on the output variance $\sigma_{\varepsilon}^{2}$. This surprising result arises because $\sigma_{\varepsilon}^{2}$ has two countervailing effects. To describe them, it is useful to recall that $m_{t+1}=\lambda m_{t}+(1-\lambda) x_{t}$, and that consumption is linear in $m$ as $c_{t}=m_{t}+a_{t}^{*}$. Hence $\sigma_{\varepsilon}^{2}$ has a positive effect on consumption volatility since it raises the variance of the market signal $x$. However, $\sigma_{\varepsilon}^{2}$ also raises the gain parameter $\lambda$ as the market puts less weight on recent observations when production is more noisy. This additional inertia in the updating rule exactly compensates the direct impact on the variance of the signal, thus leaving consumption volatility unchanged. ${ }^{5}$ In the remainder of the paper, we guarantee that expected returns are bounded by focusing on cases where $r<1$.

\footnotetext{
${ }^{5}$ Note that this finding holds because prior precision is set equal to its stationary level $\bar{\sigma}_{\theta}^{-2}$. For any other value, the conditional variance of $m$ would not anymore be independent of $\sigma_{\varepsilon}^{2}$.
} 


\section{Cycles with Linear Costs}

\subsection{Rest point}

Suppose that $g(a)=\kappa a$ with $\kappa \in(0,1)$ to ensure that effort raises output net of disutility. We rule out infinite output by imposing an upper-bound $\bar{a}$ on the feasible set $\mathcal{A}=[0, \bar{a}]$. With linear costs, optimality can only be restored through changes in the discount factor because both marginal costs and marginal returns are constant. To see why, assume that both $a_{t}^{*}$ and $a_{t+1}^{*}$ belong to the interior of $\mathcal{A}$ and substitute $g^{\prime}\left(a_{t+1}^{*}\right)$ on the right-hand side of (11) to obtain ${ }^{6}$

$$
\frac{U^{\prime}\left(a_{t+1}^{*}(1-\kappa)\right)}{U^{\prime}\left(a_{t}^{*}(1-\kappa)\right)}=\frac{\kappa}{r(1+\lambda(\kappa-1))} .
$$

Taking expectations about $a_{t+1}^{*}$ as given, $a_{t}^{*}$ adjusts until the equality above is satisfied. The equilibrium path is fully determined by the ratio of marginal costs to discounted marginal returns on the right-hand side of (13). Since this ratio is constant, all paths converge to $\bar{a}$ when it is lower than one or, conversely, converge to zero when it is higher than one. The dynamics become explicit if one uses (9) to rewrite (13) as

$$
a_{t+1}^{*}=a_{t}^{*}-\frac{1}{\gamma(1-\kappa)} \log \left(\frac{\kappa}{r(1+\lambda(\kappa-1))}\right) .
$$

The law of motion is obtained projecting $a_{t}^{*}$ on a line parallel to the 45 degree line with an intercept equal to the constant on the right-hand side of (14). As shown in Appendix A.2, Fig. 13, in the knife-edge case where the intercept is zero, i.e., when $\kappa=(1-\lambda) /\left(r^{-1}-\lambda\right)$, the dynamic map and 45 degree line coincide so that any action in $\mathcal{A}$ is a potential rest point. Besides this particular case, the action path converges to $\bar{a}$ when the intercept is positive or to zero when it is negative. Hence $a_{t}^{*}$ can be constant solely at one of the boundary of the action set.

If marginal costs are low, so that $\kappa<(1-\lambda) /\left(r^{-1}-\lambda\right)$, agents are tempted to raise their effort above that of the rest point $\hat{a}$. Hence $\hat{a}$ is sustainable only if such deviations are not feasible, that is if $\hat{a}=\bar{a}$. ${ }^{7}$ This solution is also efficient because the

\footnotetext{
${ }^{6} \mathrm{Eq}$. (13) is a particular case of the recursive incentive constraint (18) where $g(a)=\kappa a$. The posterior $m$ does not affect the ratio on the LHS of (13) because utility is CARA.

${ }^{7}$ Formally, the incentive constraint (11) does not have to hold as an equality at the bounds of the feasibility set. When $\hat{a}=\bar{a}$ and $\kappa<(1-\lambda) /\left(r^{-1}-\lambda\right)$, the RHS exceeds the LHS of (11) and the agent is constrained by the requirement that $a \leq \bar{a}$.
} 
cost parameter $\kappa<1$ while marginal productivity is one. Conversely, when marginal costs are high, so that $\kappa>(1-\lambda) /\left(r^{-1}-\lambda\right)$, reputational concerns are too weak and $\hat{a}=0$ is the only rest point.

\subsection{Cycles}

Deterministic output.-To build our intuition, we first assume that output is deterministic, i.e., $\sigma_{\varepsilon}=0$. Then $\bar{\sigma}_{\theta}^{2}=\sigma_{\nu}^{2}$ since all the uncertainty is due to the fact that the agent's type $\theta$ changes from period to period. If $\theta$ were to remain constant, each type would be revealed once and for all after the first output realization. This simplified environment enables us to isolate the impact of the discount factor. As shown in Proposition 3, the model exhibits stationary cycles of period two when agents are sufficiently patient. Moreover, two-period cycles can Pareto dominate the rest point when the latter is inefficient because effort costs are too high.

Proposition 3 Assume that (i) costs are linear in effort $(g(a)=\kappa a$ with $\kappa \in(0,1))$; (ii) $a \in[0, \bar{a}]$; and (iii) output is deterministic $\left(\sigma_{\varepsilon}=0\right)$. Then the rest point $\hat{a}=0$ is Pareto dominated by 2-period cycles in which effort oscillates between 0 and $\bar{a}$ whenever $r \in(\kappa \exp (-\gamma(1-\kappa) \bar{a}), \kappa)$.

Cycles arise because the discount factor fluctuates procyclically. High $a_{t}=\bar{a}$ entails above-normal consumption, leading to a low marginal utility $U^{\prime}\left(c_{t}-g\left(a_{t}\right)\right)$. The opposite is true next period as $a_{t+1}=0$ delivers low consumption and relatively high marginal utility $U^{\prime}\left(c_{t+1}-g\left(a_{t+1}\right)\right)$. Thus $E_{t}\left[U^{\prime}\left(c_{s}-g\left(a_{s}\right)\right) / U^{\prime}\left(c_{t}-g\left(a_{t}\right)\right)\right]$ is large today, and this justifies the higher investment in reputation building. A similar but opposite mechanism operates in the next period, making low effort optimal. The oscillations of the discount factor capture the willingness of the agent to smooth consumption. Since the benefits of her action only accrue in the following period, she finds it optimal to transfer resources from good to bad times by overinvesting.

To better understand the forces at work, it is insightful to study the incentive constraint at the upper-bound $\bar{a}$. Full effort is incentive compatible if and only if ${ }^{8}$

$$
\kappa \leq r \frac{U^{\prime}(0)}{U^{\prime}((1-\kappa) \bar{a})}=r \exp (\gamma(1-\kappa) \bar{a}) .
$$

\footnotetext{
${ }^{8}$ See proof of Proposition 4 for a derivation of (15).
} 
Reputational returns are on the right-hand side of (15), and they are increasing in effort $\bar{a}$ because it raises the ratio of marginal utilities between bad and good times. Quite intuitively, smaller oscillations become sustainable when the power of incentives is strengthened. Take for example an increase in the discount factor $\beta$. It raises $r$ as patient agents tend to be more concerned by their reputation. Hence the required wedge between marginal utilities is decreasing in $\beta$ which allows the model to generate cycles for lower values of $\bar{a}$. A similar mechanism is triggered by changes in the degree of risk aversion because the effective rate of time preference $r$ is increasing in $\gamma$. This effect is reinforced by the positive impact that $\gamma$ has on the curvature of the marginal utility function. When agents are more prudent, similar oscillations in consumption generate greater swings in the discount factor, which lowers the value of $\bar{a}$ that restores incentive compatibility.

To sum-up, the agent must be sufficiently prudent and not too impatient for cycles to emerge. Note that Proposition 3 also requires that agents are not too patient since it imposes an upper-bound on the effective discount factor $(r<\kappa)$. Although cycles can emerge even when this requirement is violated, they will not be welfare improving because, as explained before, the rest point is efficient when costs are so low that $r>\kappa .^{9}$

Stochastic output.-We now consider the full model where output is stochastic. Letting $\sigma_{\varepsilon}$ differ from 0 implies that today's action affects beliefs on-the-equilibrium path not only in the next period, but also in all future periods. The rate of decay of past observations is controlled by the parameter $\lambda$, whose value is equal to 0 when output is observable. Proposition 4 takes into account the impact of $\lambda$ by extending Proposition 3 to environments where output is noisy.

Proposition 4 Assume that (i) costs are linear $(g(a)=\kappa a$ with $\kappa \in(0,1)$ ); and (ii) $a \in[0, \bar{a}]$. There exists a unique $\underline{r}<\frac{\kappa}{1-\lambda+\lambda \kappa}$ such that the rest point $\hat{a}=0$ is Pareto dominated by 2-period cycles in which effort oscillates between 0 and $\bar{a}$ whenever $r \in$ $\left(\underline{r}, \frac{\kappa}{1-\lambda+\lambda \kappa}\right)$. The cutoff discount factor $\underline{r}$ is decreasing in $\bar{a}$ and $\gamma$.

As with deterministic output, welfare improving cycles can arise solely if the agent is sufficiently patient and prudent. We can also show that they require output to not be too noisy. Thus, although $\sigma_{\varepsilon}$ is, by the usual account, a measure of idiosyncratic uncertainty, higher $\sigma_{\varepsilon}$ does not necessarily translate into more income volatility for

\footnotetext{
${ }^{9}$ Remember that $\lambda=0$ when $\sigma_{\varepsilon}=0$.
} 
the agent. An increase in $\sigma_{\varepsilon}$ lowers the power of incentives because recent observations are less informative when realized output is a noisier measure of actual quality. This implies in turn that cycles are not sustainable if $\sigma_{\varepsilon}$ is too high.

Proposition 5 There exists a unique output variance $\bar{\sigma}_{\varepsilon}$ such that cycles of period two are never sustainable when $\sigma_{\varepsilon}>\bar{\sigma}_{\varepsilon}$. Moreover, (i) when $\kappa>r$, cycles are sustainable if and only if $\bar{a} \geq \frac{\log (\kappa / r)}{\gamma(1-\kappa)}$ and $\sigma_{\varepsilon} \in\left[0, \bar{\sigma}_{\varepsilon}\right]$; (ii) when $\kappa \leq r$, there exists an output variance $\underline{\sigma}_{\varepsilon} \in\left[0, \bar{\sigma}_{\varepsilon}\right)$ such that cycles are sustainable for all $\sigma_{\varepsilon} \in\left[\underline{\sigma}_{\varepsilon}, \bar{\sigma}_{\varepsilon}\right]$.

According to Proposition 5, a reduction in the noisiness of the technology can raise income volatility. This paradoxical behavior occurs if: (i) $\sigma_{\varepsilon}$ is initially higher than the upper-bound $\bar{\sigma}_{\varepsilon}$, so that $a_{t}^{*}$ is constant for all $t$; and (ii) the drop in $\sigma_{\varepsilon}$ is large enough to ensure that the new value is smaller than $\bar{\sigma}_{\varepsilon}$, so that effort now cycles between 0 and $\bar{a}$. Then the cross-sectional and conditional variance of income will be negatively correlated with $\sigma_{\varepsilon}$. As mentioned in the introduction, this relates to the evidence on rising inequality in SPGBW.

The variance of income increments from period to period is given by ${ }^{10}$

$$
\begin{aligned}
\operatorname{Var}\left(m_{t+1}+a_{t+1}^{*}-\left(m_{t}+a_{t}^{*}\right)\right) & =(1-\lambda)^{2}\left(\sigma_{\varepsilon}^{2}+\bar{\sigma}_{\theta}^{2}\right)+\operatorname{Var}\left(a_{t}^{*}\right) \\
& =\sigma_{\nu}^{2}+\operatorname{Var}\left(a_{t}^{*}\right) .
\end{aligned}
$$

The variance of $a_{t}^{*}$ is obviously equal to 0 at the rest point. It jumps to $\bar{a}^{2} / 4$ when $\sigma_{\varepsilon}$ crosses the $\bar{\sigma}_{\varepsilon}$ threshold from above and effort starts to cycle. Such a drop in output uncertainty will therefore increase the conditional volatility of income. To characterize its impact on the cross-sectional variance, we must first ensure that a stationary distribution of types exists. The simplest way to do so is to assume that agents randomly exit the market with probability $\delta$ per period. Since each agent's productivity is a geometrically distributed random variable and an additional period of life adds $\sigma_{\nu}^{2}$ to the variance of $m$, the time-invariant distribution of types $\Upsilon(m)$ is a mixture of normal distributions with the following cross-sectional variance

$$
\operatorname{Var}\left(m_{t}+a_{t}^{*}\right)=\sigma_{\nu}^{2} \sum_{t=1}^{\infty} t \delta(1-\delta)^{t}+\operatorname{Var}\left(a_{t}^{*}\right)=\sigma_{\nu}^{2} \frac{1-\delta}{\delta}+\operatorname{Var}\left(a_{t}^{*}\right)
$$

Hence $\sigma_{\varepsilon}$ lowers income variance if it crosses the $\bar{\sigma}_{\varepsilon}$ threshold and triggers a switch from the cycling regime to the rest point, as depicted in Fig. 1. The impact of the

\footnotetext{
${ }^{10}$ See the proof of Proposition 2 in the Appendix for a derivation of the second equality.
} 


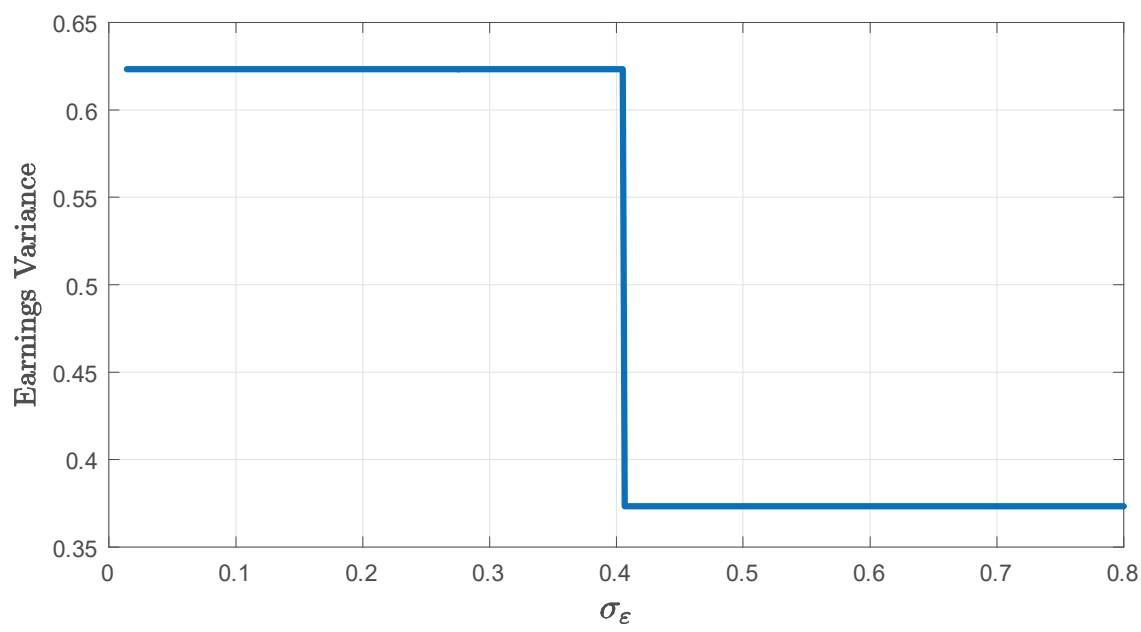

Figure 1: CROSS-SECTIONAL EARNINGS VARIANCE AS A FUNCTION OF $\sigma_{\varepsilon}$. Notes: $\gamma=3, r=.8, \kappa=.9, \sigma_{v}=.4, \delta=.3, \mathcal{A}=[0,1]$.

other volatility parameter $\sigma_{\nu}$ is less intriguing because it strengthens the power of incentives. It is therefore positively correlated with income variance since a switch to the cycling regime will be triggered by an increase in $\sigma_{\nu}$, as opposed to a decrease in $\sigma_{\varepsilon}$. Note that $\sigma_{\nu}$ also directly increases the volatility of income by augmenting the variance of income increments from period to period (see eq. (16)). As a result $\sigma_{\nu}$ raises the discount factor $r$, which further supports the emergence of cycles.

\section{Cycles with Convex Costs}

We now generalize our setup and show that cycles continue to emerge when disutility is strictly convex in effort. If $U(c, a)=U(c-g(a))$ and if $g^{\prime}(0)=0$, the incentive constraint (10) always admits an interior solution for $a^{*}$, and we can substitute in the expression of $g^{\prime}\left(a_{t+1}^{*}\right)$ on the right-hand side of (10) to obtain a recursive condition

$$
g^{\prime}\left(a_{t}^{*}\right)=\beta E_{t}\left[\frac{U^{\prime}\left(c_{t+1}-g\left(a_{t+1}^{*}\right)\right)}{U^{\prime}\left(c_{t}-g\left(a_{t}^{*}\right)\right)}\right]\left(1-\lambda+\lambda g^{\prime}\left(a_{t+1}^{*}\right)\right) .
$$

The recursive incentive constraint highlights that increasing $a_{t}$ has two benefits:

(i) It raises $t+1$ earnings by $(1-\lambda)$, and

(ii) it enables the agent to reduce her effort in period $t+1$ by $\lambda$ in order to undo the deviation, thereby restoring the reputation associated to the equilibrium path in 
$t+2$ and beyond.

Both benefits are converted into today's utils through multiplication by the stochastic discount factor $\beta E_{t}\left[U^{\prime}\left(c_{t+1}-g\left(a_{t+1}^{*}\right)\right) / U^{\prime}\left(c_{t}-g\left(a_{t}^{*}\right)\right)\right]$. When the utility function is CARA, (18) is equivalent to

$$
g^{\prime}\left(a_{t}^{*}\right)=r \exp \left(\gamma\left[a_{t}^{*}-g\left(a_{t}^{*}\right)-\left(a_{t+1}^{*}-g\left(a_{t+1}^{*}\right)\right)\right]\right)\left(1-\lambda+\lambda g^{\prime}\left(a_{t+1}^{*}\right)\right) .
$$

Setting $a_{t+1}^{*}$ equal to $a_{t}^{*}$ yields the rest point solution

$$
\hat{a}=g^{\prime-1}\left(\frac{1-\lambda}{r^{-1}-\lambda}\right) .
$$

Efficient effort would require instead that $a=g^{-1}(1)$. Thus, when $r$ is smaller than one, $\hat{a}<g^{\prime-1}(1)$ and effort at the rest point is suboptimal because costs are paid upfront, whereas reputational benefits accrue slowly over time. This delay explains why the gap between efficient and actual effort is larger when the agent is more impatient.

Stability of the rest point.-Under risk neutrality, the rest point is always unstable. Any other action than $\hat{a}$ generates diverging trajectories, ${ }^{11}$ hence the unique deterministic equilibrium requires that $a_{t}^{*}=\hat{a}$ for all $t$. By contrast, the rest point can be locally stable when the agent is risk averse. Then, for any initial action $a_{0}$ in the neighborhood of $\hat{a}$, there exists a deterministic path that converges back to $\hat{a}$. The condition under which equilibrium multiplicity arises is laid-out in Proposition 6.

Proposition 6 Assume that $g(a)$ is strictly convex with $g^{\prime}(0)=0$. Then the rest point $\hat{a}$ is locally stable if and only if

$$
\left(\frac{1-\lambda+2 \lambda g^{\prime}(\hat{a})}{1-\lambda+\lambda g^{\prime}(\hat{a})}\right) \frac{g^{\prime \prime}(\hat{a})}{g^{\prime}(\hat{a})}<2 \gamma\left(1-g^{\prime}(\hat{a})\right)
$$

Equation (20) depends on the action $\hat{a}$ at the rest point, and so only implicitly describes the parameter values under which multiple equilibria arise. We can nonetheless easily establish a couple of observations. First, when the coefficient of absolute risk aversion $\gamma$ goes to zero, condition (20) is violated and the rest point is unstable. ${ }^{12}$ Second, if $\hat{a}$ is efficient, the rest point is unstable. To see why, note that the impact of

\footnotetext{
${ }^{11}$ See Fig. 2 for an illustration of the model's dynamics under risk neutrality.

${ }^{12}$ This is not obvious from (20) because $\gamma$ affects $\hat{a}$ through its impact on $r$. However, since $r$ converges to $\beta$ when $\gamma$ goes to zero, $\lim _{\gamma \rightarrow 0} \hat{a}(\gamma)=g^{\prime-1}\left((1-\lambda) /\left(\beta^{-1}-\lambda\right)\right)$. Hence the LHS of $(20)$ converges to a positive value, whereas the RHS goes to zero, and so (20) never holds in the limit.
} 
$\gamma$, as measured by the term on the right hand side of (20), is proportional to $1-g^{\prime}(\hat{a})$. Thus risk aversion is irrelevant when $g^{\prime}(\hat{a})=1$, that is when the action is efficient. Intuitively, the curvature of the utility function matters only to the extent that changes in $a$ affect utility. Benefits and costs are set equal at the first best and a marginal increase in effort leaves utility unchanged. However, we have seen that $\hat{a}$ is inefficient whenever the agent discounts the future. Then raising $a$ lowers her marginal utility, thus making it attractive to provide more effort today in order to raise tomorrow's consumption. This is why an increase in effort is followed by a drop and a return to the rest point if the curvature of the utility function is high enough.

Cycles.-When the rest point is locally stable, there is a continuum of deterministic equilibria whose paths start in the vicinity of the stationary solution and gradually converge to it. Hence they only temporarily differ from the solution already studied in the career concerns literature. But the model can also generate more complex behaviors with persistent oscillations. We now focus on the simplest equilibria in such class, namely deterministic cycles of period two.

Proposition 7 When the costs function is quadratic, i.e., $g(a)=a^{2} / 2$, deterministic 2-period cycles are sustainable whenever the rest point is locally stable.

The reason why cycles are sustainable is the same as in the model with linear costs: Procyclical fluctuations in the discount factor make it optimal to work hard when consumption is high and to reduce effort when consumption is low. This mechanism is closely related to the one that renders the rest point locally stable, and Proposition 7 shows that the two phenomena are indeed concomitant when disutility is quadratic in effort.

Inspecting the phase portrait generated by the incentive constraint (18) helps one understand how cycles arise. We highlight the impact of risk aversion by also reporting the model's dynamics when agents are risk neutral. Then, as shown in the lefthand panel of Fig. 2, the mapping between $a_{t}^{*}$ and $a_{t+1}^{*}$ is linear with a slope greater than one. Hence the rest point $\hat{a}$ is the only effort level that does not generate diverging action paths.

We introduce risk aversion in the right-hand panel of Fig. 2. The dynamic map solves the recursive equation (19) with a quadratic cost function $g(a)=a^{2} / 2$, and is therefore equivalent to

$$
\exp \left(-\gamma\left(a_{t}^{*}-a_{t}^{* 2} / 2\right)\right) a_{t}^{*}=r \exp \left(-\gamma\left(a_{t+1}^{*}-a_{t+1}^{* 2} / 2\right)\right)\left[1-\lambda+\lambda a_{t+1}^{*}\right]
$$



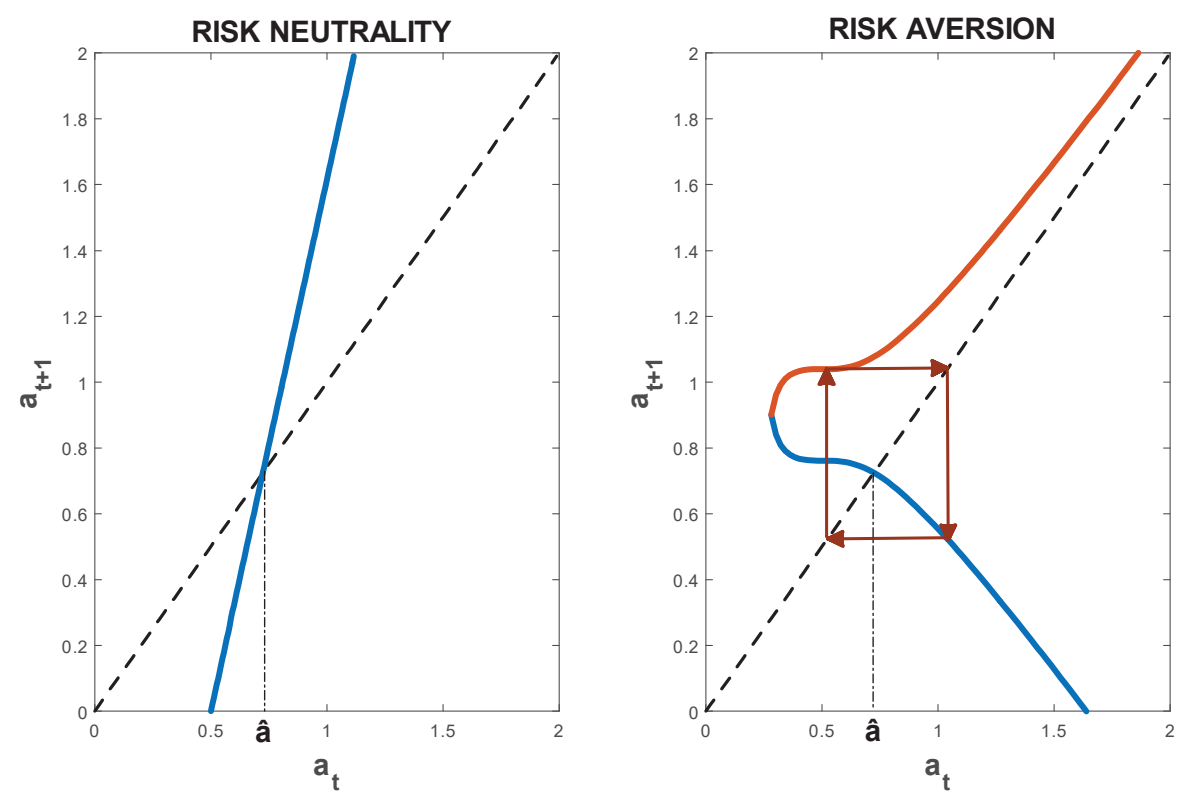

Figure 2: Phase Portraits With LineAR AND CARA Utility.

Notes: $\lambda=.4, \beta=.9, \gamma=4, g(a)=a^{2} / 2$.

Let us first explain why small values of $a_{t}^{*}$ can never be incentive compatible. Letting $a_{t}^{*}$ go to zero, we see that the expression on the left hand side of (21) also converges to zero. By contrast, the expression on the right hand side of (21) has a positive minimum. By continuity, there always exists an $\underline{a}>0$ such that (21) does not hold whenever $a_{t}^{*} \in[0, \underline{a}]$. In economic terms, there is no expectation about $a_{t+1}^{*}$ that sustains an effort level smaller than $\underline{a}$. Intuitively, the dynamic map in Fig. 2 is empty close to the origin because today's marginal loss converges to 0 whereas tomorrow's marginal returns are bounded from below by $1-\lambda$.

Besides this empty interval, we see that, instead of the one-to-one mapping prevailing under risk neutrality, the dynamic map under risk aversion is a correspondence which associates a pair of incentive compatible $a_{t+1}^{*}$ to any $a_{t}^{*}$. The incentive constraint is satisfied by two different $a_{t+1}^{*}$ because tomorrow's effort shifts the discount factor and the marginal costs in opposite directions. Increasing $a_{t+1}^{*}$ raises tomorrow's consumption, which lowers the discount factor and counteracts the increase in marginal costs $g^{\prime}\left(a_{t+1}^{*}\right)$. When the elasticity of the discount factor with respect to $a_{t+1}^{*}$ is higher than that of the marginal costs, it is possible to perturb a pair of sustainable actions and restore incentive compatibility by adjusting $a_{t+1}^{*}$ until its 

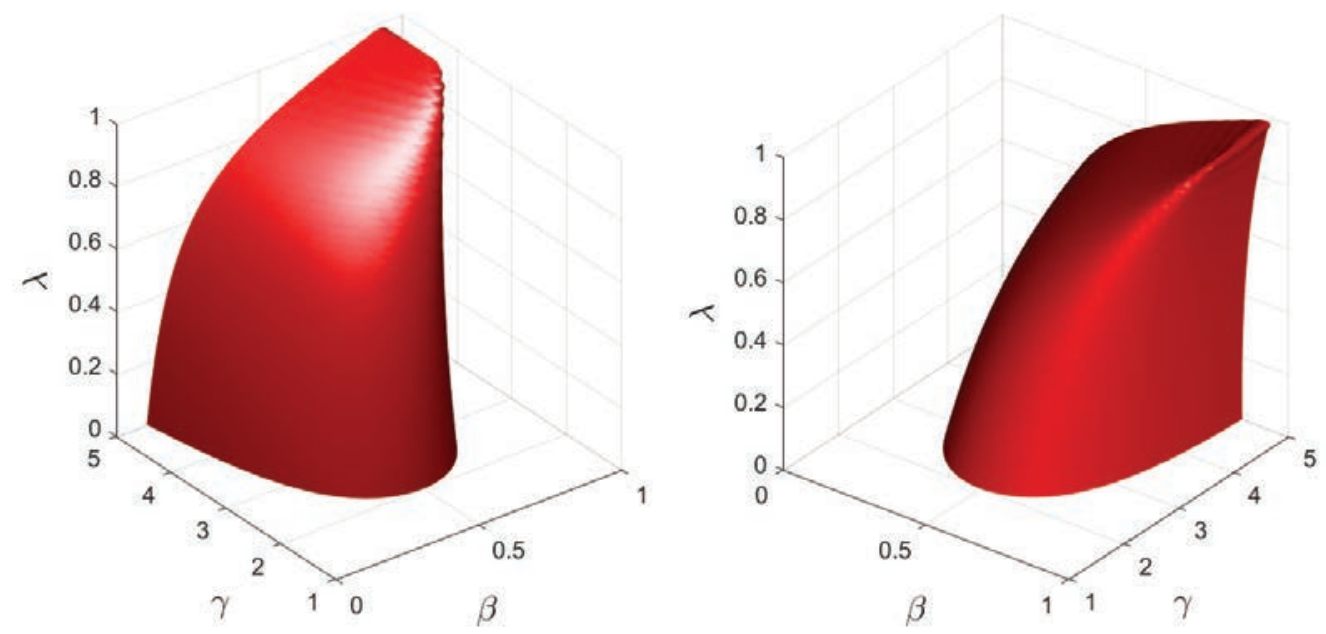

Figure 3: Stability of the Rest Point $\hat{a}$ as a Function of $\gamma, \beta$ And $\lambda$.

effect on the discount factor offsets the change in marginal costs.

Effort settles down at the rest point $\hat{a}$ when the expectation-formation mechanism always selects the lower branch of the phase portrait. By contrast, 2-period cycles are regime switching equilibria whose actions oscillate between the upper and lower branch.

Stability region.-Equation (20) enables us to identify the region with multiple equilibria. The rest point is locally stable and 2-period cycles arise whenever the parameters fall inside the surface depicted in Fig. 3. We use two different angles to depict the stability region because its shape is strongly asymmetric.

Looking first at the discount factor $\beta$, we see that it has an ambiguous impact as cycles emerge only for intermediary values of $\beta$. When $\beta$ goes to 0 , so does the rest point $\hat{a}$ because the agent is not anymore concerned by her reputation. Hence the marginal disutility of effort $g^{\prime}(\hat{a})$ also converges to 0 , and the stability condition (20) is always violated. Reputational returns are so low that an increase in today's effort can be incentive compatible solely if the marginal disutility of effort in the next period increases more than proportionally. Thus the slope of the relationship between $a_{t}^{*}$ and $a_{t+1}^{*}$ is greater than 1 when evaluated at $\hat{a}$, which ensures that the rest point is 
locally unstable. Conversely, as $\beta$ goes to 1 , $\hat{a}$ converges to the efficient level of effort which, as explained above, is always locally unstable.

Comparing the impact of the coefficient of risk aversion $\gamma$ in the right and lefthand panels of Fig. 3, we notice that $\gamma$ has a stronger effect on the stability region when $\beta$ is low. This is because $\gamma$ does not only increase risk aversion but also renders the agent more prudent. As explained before, the precautionary motive increases the level of effort at the rest point. When $\hat{a}$ is already close to its efficient level because $\beta$ is high, as in the right-hand panel of Fig. 3 , then the precautionary channel counteracts the risk aversion channel. By contrast, when $\beta$ is low, as in the left-hand panel of Fig. 3 , the risk aversion channel dwarfs the precautionary channel, so that raising $\gamma$ makes it much more likely for cycles to be sustainable.

The last variable included in Fig. 3 is the gain parameter $\lambda$ which controls the inertia of beliefs. Remember that varying $\lambda$ is equivalent to adjusting the output variance $\sigma_{\varepsilon}^{2}$, with $\sigma_{\varepsilon}^{2}$ going from 0 to infinity as $\lambda$ goes from 0 to $1 .{ }^{13}$ As already discussed in Section 3, decreasing $\lambda$ makes it more likely that cycles arise by strengthening the power of incentives. However, it also increases the value of the rest point, which may counteract the direct impact of $\lambda$ when the rest point is close to its efficient level. As was the case for $\gamma$, this countervailing force explains why the stability region is much less sensitive to changes in $\lambda$ in the right-hand panel of Fig. 3, where $\beta$ and thus $\hat{a}$ are both close to one.

Output volatility and income variance.-Studying the model with linear costs, we proved that a reduction in the noisiness of the technology may raise income variance. A similar conclusion can be numerically established in the model with convex costs. For instance, taking the parameters in Table 1 and varying the value of $\sigma_{\varepsilon}$, we indeed find that income variance and output volatility can be negatively correlated when effort fluctuates endogenously.

The upper panel of Fig. 4 reports effort in the high and low states of 2-period cycles along with its value at the rest point. Cycles are sustainable when incentives are strong enough. Hence they arise when $\lambda$, and consequently $\sigma_{\varepsilon}$, are below a certain threshold. ${ }^{14}$ The cross-sectional variance of income increases as $\sigma_{\varepsilon}$ crosses this

\footnotetext{
${ }^{13}$ By contrast, the variance of types $\sigma_{\nu}^{2}$ affects two parameters at once. Raising $\sigma_{\nu}^{2}$ leads to a decrease in $\lambda$ as well as an increase in $r$, which is equivalent to raising $\beta$ in Fig. 3. In general, these two adjustments tend to reinforce each other, thus making it more likely that cycles are sustainable.

${ }^{14}$ Note that Fig. 3 shows that it is not always possible to generate cycles by lowering $\lambda$, especially when $\beta$ is close to one so that the rest point is not too far from its efficient level.
} 

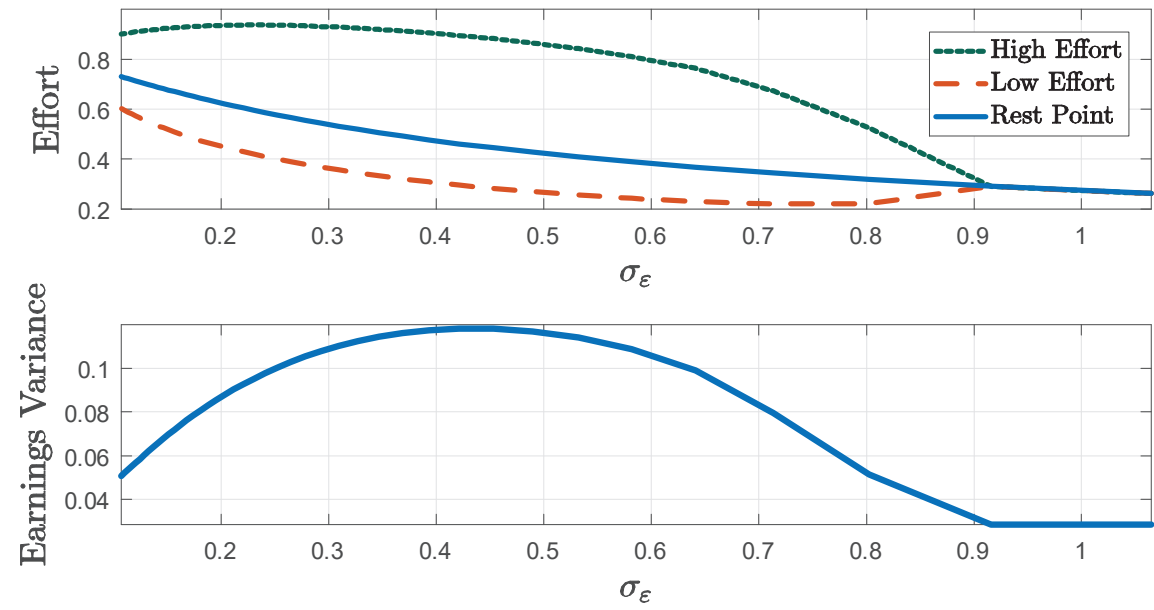

Figure 4: EquiLibrium EFFORT AND EARNingS VARIANCE AS A FUNCTION OF $\sigma_{\varepsilon}$. Notes: Other parameters reported in Table 1.

bifurcation point because effort is not anymore the same across all agents. The bottom panel is the analog of Fig. 1; this experiment confirms that an increase in income variance can be caused by a reduction in the volatility of the underlying shocks. Moreover, we see that the calibrated value of $\sigma_{\varepsilon}$ in Table 1 falls well within the region where cycles are sustainable. This again relates to the evidence on rising inequality in SPGBW.

\section{Earnings Persistence}

\subsection{Deterministic cycles}

Parametrization.-We have established that reputation cycles emerge when the riskaversion and the patience of the agent belong to some intermediate range. To see whether these two requirements are likely to be met in practice, we use micro data to pin down the parameters.

We now assume that agents are workers who maximize their expected utility, and that $a$ is a worker's hidden labor. We focus on quadratic disutility so that $g(a)=a^{2} / 2$. The model then has five parameters $\left\{\gamma, \beta, \delta, \sigma_{\varepsilon}, \sigma_{\nu}\right\}$. For the yearly discount factor, we set $\beta=0.95$. The exit rate $\delta$ determines the average length of a worker's career. Assuming that reputation is occupation specific, we target Kambourov and Manovskii's (2008) finding that occupational mobility at the one-digit level averaged $13 \%$ per year 
in PSID data over the 1968-1997 period.

The two variance parameters $\left\{\sigma_{\varepsilon}, \sigma_{\nu}\right\}$ are chosen to match the speed of employer learning and the labor wedge. We use $\tau$ to denote the labor wedge and define it as the gap between the marginal product of labor (MPN) and the marginal rate of substitution (MRS), so that $\tau \equiv 1-\mathrm{MRS} / \mathrm{MPN}$. The labor wedge measures the extent to which agents underinvest in their reputation. In the absence of distortions, $\tau=0$ because both MRS and MPN are equal to the wage. At the rest point of our model, however, labor supply is inefficiently low and the MRS is lower than the wage. Hence the labor wedge is positive and equal to ${ }^{15}$

$$
\hat{\tau}=\frac{r^{-1}-1}{r^{-1}-\lambda} \in(0,1),
$$

where $\lambda$ and $r$ are defined in (7) and (12). We write $\hat{\tau}$ for the wedge when labor supply is at the rest point so that $a=\hat{a}$. We set $\hat{\tau}=0.4$ to match the average labor wedge in the US over the last fifty years, as estimated by Shimer (2009).

For our second moment, we target the frequency at which the market updates its beliefs, i.e., the speed with which information about the agent's performance leaks out to the general public. Its value has been estimated by Lange (2007) in his empirical analysis about the speed of employer learning. Using AFQT scores to infer ability, Lange's estimates of the learning speed center around $\lambda=.75 .{ }^{16}$

Together with Eqs. (7), (22), these estimates for $\lambda$ and $\tau$ enable us to to pin down $\sigma_{\varepsilon}$ and $\sigma_{\nu}$ conditional on $\gamma$. The upper panel of Fig. 5 shows that reputation cycles are consistent with our calibration strategy whenever

$$
\gamma \geq 3.4
$$

The amplitude of the oscillations increases with $\gamma$, as greater fluctuations are required to offset the increase in the curvature of the utility function. The lower panel of Fig. 5 shows that effort at the rest point is always smaller than average effort in the cycling

\footnotetext{
${ }^{15}$ The wage can be normalized to one without loss of generality. The technology being linear in supplied labor $a$, the MPN is also equal to one and so the labor wedge at the rest point is equal to $\hat{\tau}=1-\mathrm{MRS} / \mathrm{MPN}=1-g^{\prime}(\hat{a})=1-\frac{1-\lambda}{r^{-1}-\lambda}$, i.e., (22).

${ }^{16}$ Lange (2007) uses $K_{1}$ to denote the speed of learning parameter and defines it on page 11, eq. (11). Comparing its expression with that of $\lambda$ in (7), one sees that $K_{1}=1-\lambda$. Even though learning in Lange's model is non-stationary, since types remain constant over time in his model, the similarity between $K_{1}$ and $\lambda$ holds because $K_{1}$ captures the speed of learning during the first year of a worker's career.
} 
regime, thus substantiating our claim that financial constraints may incentivize investment in reputation. Although $\gamma$ is a CARA-utility parameter, we can convert (23) into a statement about CRRA utility as follows: By adjusting average ability $\theta_{0}$, we can normalize average consumption and output to unity at the rest point. ${ }^{17}$ Then $\gamma$ also corresponds to the average coefficient of relative risk aversion. Picking $\gamma=4$, while setting $\sigma_{\varepsilon}=.2255$ and $\sigma_{\nu}=.0651$, ensures that we exactly match our labor wedge and speed of learning targets. Our choice of parameters is summarized in Table 1.
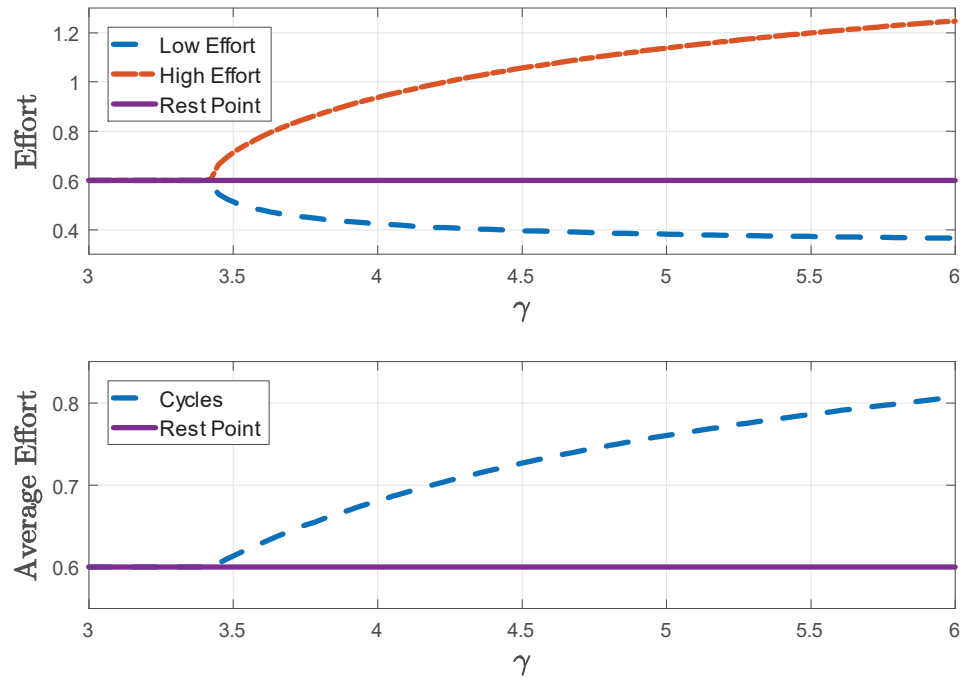

Figure 5: Deterministic CyCles AS a Function of the Coefficient of Risk AverSION $\gamma$.

Notes: Baseline parameters reported in Table 1.

Earnings persistence.-We now explain why reputation cycles generate earnings profiles with large shocks that are less persistent than small shocks, as documented in GKOS and ABB. To relate mean reversion to the size of the shocks, we follow GKOS in reporting today's earnings changes against the expected value of future earnings changes $k$ years from now, for different values of $k$. The resulting impulse response functions are depicted in Fig. 6. They measure the fraction of each shock that has mean-reverted. The points that are on the horizontal line indicate perfect persistence of today's shocks, whereas those that are on the 45 degrees line indicate full mean

\footnotetext{
${ }^{17}$ More precisely, setting $\theta_{0}=0.4$ ensures that consumption at the rest point is on average equal to 1 since $E[\hat{c}]=\theta_{0}+\hat{a}=\theta_{0}+1-\hat{\tau}$, and $\hat{\tau}$ has been set equal to 0.4 to match the evidence in Shimer (2009).
} 
Table 1: Calibrated Parameters

\begin{tabular}{|c|c|c|c|}
\hline \multicolumn{4}{|c|}{ DIRECTLY CALIBRATED PARAMETERS } \\
\hline PARAMETER $^{\dagger}$ & INTERPRETATION & SOURCE & \\
\hline$\beta=.95$ & Discount factor & Standard & \\
\hline$\gamma=4$ & Coefficient of risk aversion & Standard & \\
\hline$\theta_{0}=.4$ & Mean initial ability & Normalize consumption $\hat{c}=1$ & \\
\hline$\delta=.13$ & Career switching rate & Kambourov et al. (2008) & \\
\hline \multicolumn{4}{|c|}{ INDIRECTLY CALIBRATED PARAMETERS } \\
\hline PARAMETER $^{\dagger}$ & INTERPRETATION & TARGET & SOURCE \\
\hline$\sigma_{\varepsilon}=.2255$ & Output noise & Labor wedge $\hat{\tau}=.4$ & Shimer (2009) \\
\hline$\sigma_{\nu}=.0651$ & Type volatility & Speed of learning $\lambda=.75$ & Lange (2007) \\
\hline \multicolumn{4}{|c|}{ INDIRECTLY CALIBRATED PARAMETERS OF STOCHASTIC MODEL } \\
\hline PARAMETER $^{\dagger}$ & INTERPRETATION & TARGET & SOURCE \\
\hline$\sigma_{\xi}=.11$ & Volatility observable productivity & Std. deviation log earnings changes & Guvenen et al. (2015) \\
\hline$\alpha=.7$ & Switching probability & Persistence log earnings growth & Guvenen et al. (2015) \\
\hline
\end{tabular}

Notes. ${ }^{\dagger}$ When applicable, parameter values are for yearly frequency.

reversion. ${ }^{18}$

We first report in the left-hand panel of Fig. 6 the impulse responses when effort is at the rest point. Then earnings shocks, whatever their sizes, are fully persistent because beliefs, and thus wages, follow a martingale process. We introduce reputation cycles in the right-hand panel of Fig. 6. Large shocks are now driven by changes in effort, with cycles considerably widening the range of income fluctuations. Focusing on horizons that are even $(k=1,3,5)$, large shocks are completely transitory because they follow the agents' efforts whose values oscillate from one period to the next.

\subsection{Three fit-improving extensions}

The following three extensions improve the model's fit to the impulse-response evidence in GKOS.

1. Observed characteristics: Given that some output characteristics may be observable, we add a state $p_{t}$ such that the output of a worker with $t$ years of occu-

\footnotetext{
${ }^{18}$ The impulse response functions are estimated using a simulated panel dataset made of 100 waves, each one of them containing 5.000 workers. We delete the first 50 waves to limit the impact of the initial conditions, thus basing our estimates on 500.000 observations.
} 

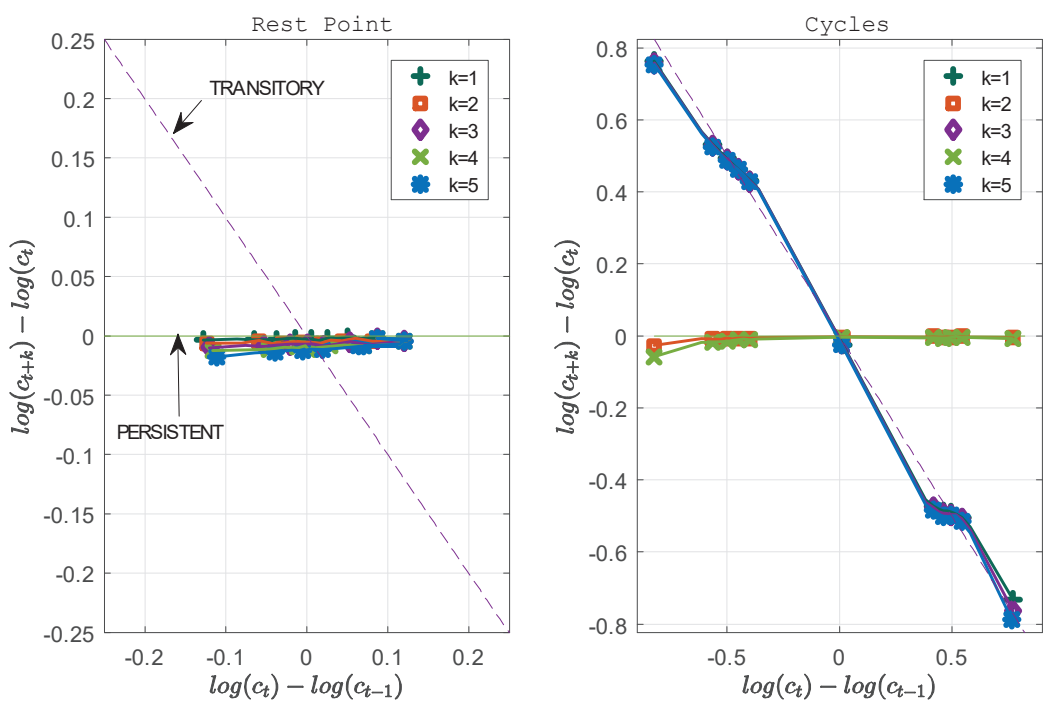

Figure 6: IMPULSE RESPONSES.

Notes: Using the parameters in Table 1, we generate a balanced panel dataset of 50 waves, with 5.000 workers per wave. For each period, we assign workers into 10 groups based on their current logearnings growth. Then, for workers in each group, we compute the average of log k-year earnings growth, i.e., $E\left[\log \left(c_{t+k} / c_{t}\right) \mid \log \left(c_{t} / c_{t-1}\right)\right]$ for $k=1,2,3,4,5$.

pational experience reads

$$
y_{t}=\underbrace{\theta_{t}+a_{t}+\varepsilon_{t}}_{\text {Unobservable }}+\underbrace{p_{t}}_{\text {Observable }} \text {, }
$$

where

$$
p_{t}=p_{t-1}+\xi_{t}, \text { with } \xi_{t} \sim \mathcal{N}\left(0, \sigma_{\xi}^{2}\right) \text { and } p_{1}=0 .
$$

Since $p_{t}$ is persistent, it drops out of the incentive constraints for the same reason that $m_{t}$ does.

2. Some workers can borrow and lend: Cycling equilibria arise only if agents cannot borrow and lend. According to Kaplan, Violante and Weidner (2014) only one third of agents are so constrained. Hence we assume that two thirds of workers can smooth their income and implement the rest point solution since that is the only Markovian equilibrium that exists in that case. This adds no new parameters.

3. Stochastic cycles: Our model exhibits a significant departure from GKOS data 
as the degree of mean reversion dramatically changes when the forward horizon $k$ goes from even to odd. Whenever $k$ is odd, shocks are fully persistent because effort reverts to its original value. This suggests that, in order to reproduce the data, one needs to consider cycles whose lengths are not deterministic.

Thus we allow agents to switch stochastically, instead of deterministically, between periods of low and high effort. Signals that raise $a_{t}$ are "good news" because they bring the worker closer to first best, signals that lower $a_{t}$ are bad news. ${ }^{19}$ We continue to focus on action paths with two levels only, but assume that, at each level, effort remains constant with probability $\alpha$, and changes state with the complementary probability. This lowers the discount factor but otherwise leaves incentives as before; details are in Appendix A.4. One coordination device may be task assignment as in Bar-Isaac and Hörner (2014) - assignment to a demanding task indicating an expectation that effort will be high, and vice versa.

\subsection{Extended calibration}

We keep the baseline parameters reported in Table 1, and select the values of the two new parameters $\left\{\alpha, \sigma_{\xi}\right\}$ to minimize the distance between a set of moments predicted by the model and their empirical counterparts. We target the estimates in GKOS for the dispersion of log-earnings growth and the persistence of earning shocks. ${ }^{20}$ As reported in Table 1, we obtain the best fit when $\alpha=.7$ and $\sigma_{\xi}=.11$.

Distribution of earnings growth.-The density of earnings changes predicted by the model, and the one observed in GKOS data, are reported in Fig. 7. We also include a normal density with the same standard deviation as in the data. Fig. 7 shows that the simulated and empirical densities display high kurtosis since they have much

\footnotetext{
${ }^{19}$ This relates to the good-news and bad-news literature. By contrast to our signals, Board and Meyer-ter-Vehn (2013), e.g., have endogenous signals to which effort can respond.

${ }^{20}$ More precisely, we split the empirical moments into two vectors: $\hat{\mathbf{m}}_{1}$ which contains the estimated standard deviation of log-earnings growth, and $\hat{\mathbf{m}}_{2}$ which contains the average persistence for the first and last five percentiles of earnings shocks. Average persistence is computed over four and five years so as to match its value when the horizon is odd or even. Using $\mathbf{m}_{i}(\boldsymbol{v})$ to denote the simulated moments generated by the vector of parameters $\boldsymbol{v} \equiv\left\{\alpha, \sigma_{\xi}\right\}$, we compute the quadratic distances $d_{i}(\boldsymbol{v}) \equiv\left(\mathbf{m}_{i}(\boldsymbol{v})-\hat{\mathbf{m}}_{i}\right) \cdot\left(\mathbf{m}_{i}(\boldsymbol{v})-\hat{\mathbf{m}}_{i}\right)^{\prime}$ for $i=1,2$. Our calibrated vector $\hat{\boldsymbol{v}}$ solves $\hat{\boldsymbol{v}}=$ $\arg \min _{v \in[0,1] \times \mathbb{R}_{+}}\left\{\left(d_{1}, d_{2}\right) \cdot W \cdot\left(d_{1}, d_{2}\right)^{\prime}\right\}$, where the weighting matrix $W$ is a diagonal matrix with the squares of the data targets on the diagonal - i.e., $W_{i i}=1 / \hat{\mathbf{m}}_{i}^{2}$ - and zero for all other entries. This procedure ensures that our objective puts the same weight on the dispersion of log-earnings growth as on the persistence of earning shocks.
} 


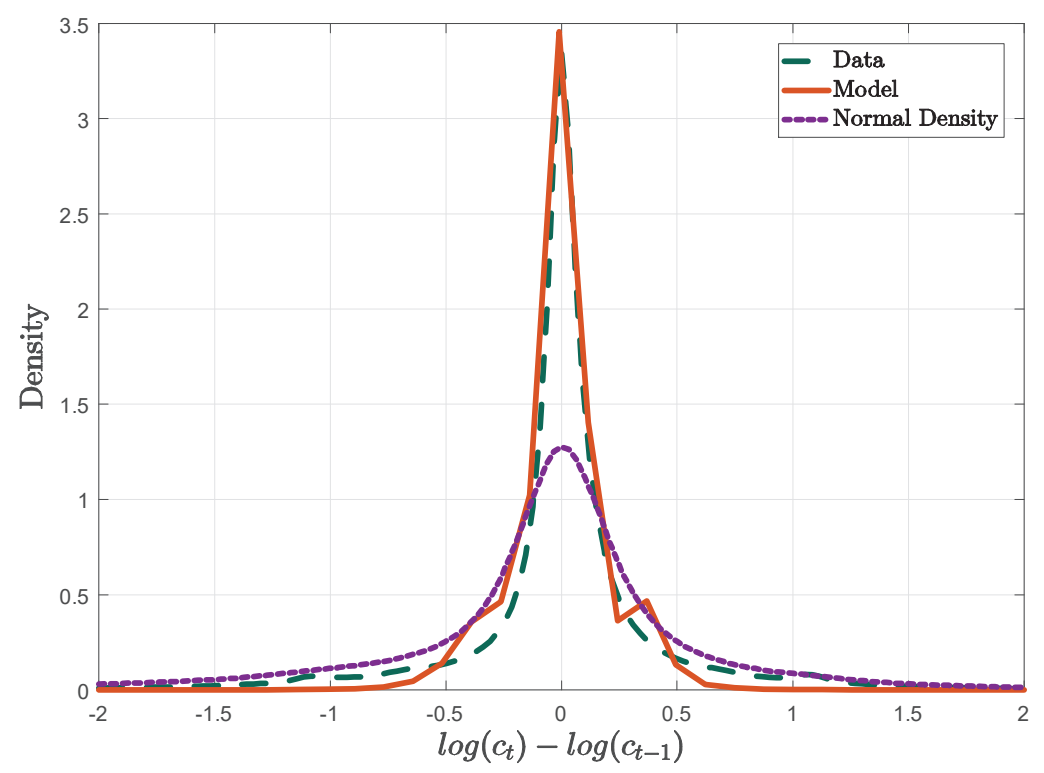

Figure 7: Histogram OF LOG EARNings CHANGES.

Notes: Data and Normal distribution are from Guvenen et al. (2009). Model's simulation based on parameters in Table 1.

sharper peaks than the normal distribution. We match the shape of the empirical peak almost perfectly. In our model, the extra kurtosis is generated by reputation cycles because the two other sources of income shocks, namely changes in reputation and observable productivity, are independently drawn from normal distributions. The impact of reputation cycles is noticeable on the shoulders of the simulated distribution, that is in the regions around the positive and negative standard deviations, where the model generates more mass than in the data.

Persistence of earnings changes.-The impulse responses of the extended model are reported in Fig. 8. They are now consistent with GKOS. The persistence of earnings changes is clearly nonlinear. Small shocks are very persistent because they result from changes in reputation or observable productivity, which are permanent by assumption. By contrast, large shocks are driven by reputation cycles that tend to revert over time. But their degree of mean reversion is not anymore fully determined by the forward horizon. Adding a stochastic switching rate $(\alpha=.7)$ smooths the impulse responses, as only $70 \%$ of saving-constrained agents adjust their effort from one period to the next.

Although the introduction of stochastic cycles ensures that the impulse responses 


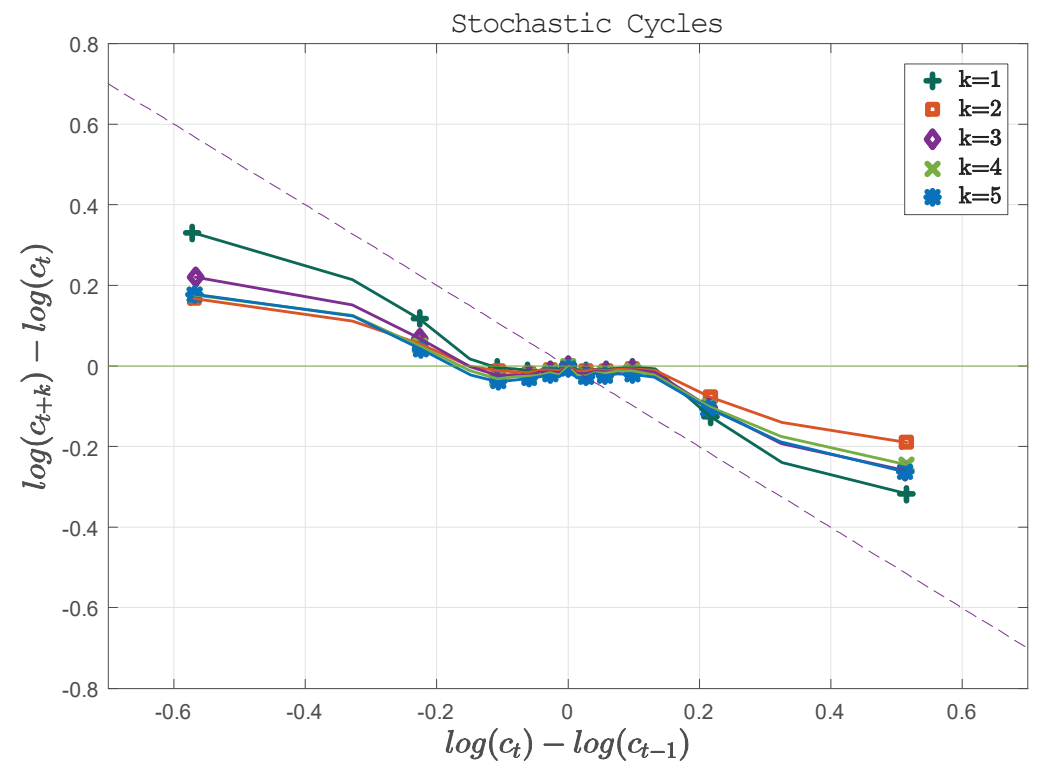

Figure 8: Impulse Responses With StOchastic CyCles.

Notes: Parameters reported in Table 1.
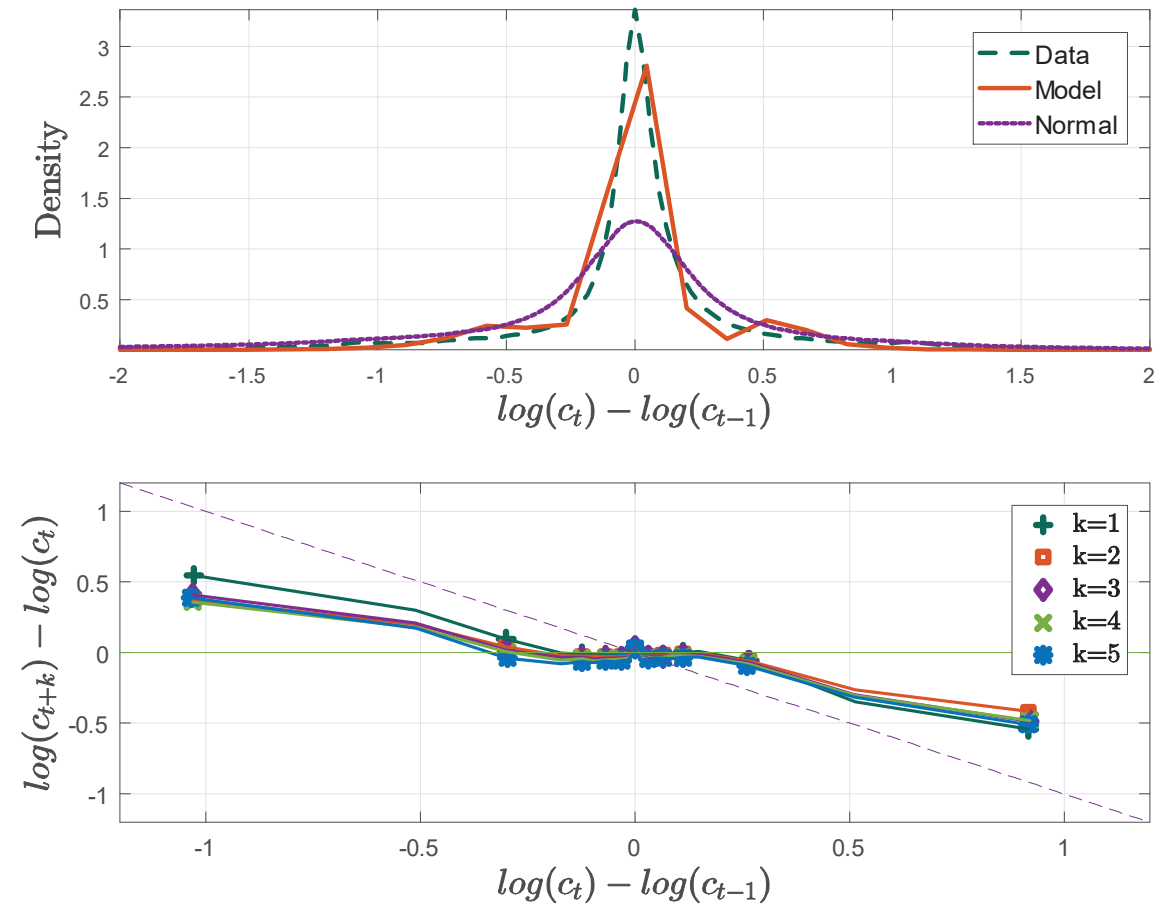

Figure 9: Histogram of Log EARnings Changes and Impulse Responses with STOCHASTIC CYCLES AND $\gamma=5$.

Notes: $\gamma=5, \alpha=.63, \sigma_{\varepsilon}=.18, \sigma_{\nu}=.052, \sigma_{\xi}=.09$, other parameters reported in Table 1 . 
are broadly similar to those reported in GKOS, the potential size of shocks is smaller in our simulations than in the data. ${ }^{21}$ Hence reputation cycles cannot account for all unusual events, especially large negative drops in earnings. To some extent, this was expected since our model does not take into consideration the risk of nonemployment. It is, however, possible to increase the size of shocks by raising the coefficient of risk aversion $\gamma$. Setting for instance $\gamma$ equal to 5 instead of 4 , and recalibrating the model, yields Fig. 9. The distribution of log-earnings changes remains close to its empirical counterpart. Raising $\gamma$ widens the amplitude of cycles which creates the following trade-off: On the one hand, the model now matches more closely the fatness of the tails; on the other hand, it slightly underestimates the size of the spike at 0 . As for the impulse responses reported in the lower panel of Fig. 9, they have a similar shape than in the benchmark calibration. The only noticeable difference is that wider cycles translate into a larger range of earnings shocks.

Impulse responses by income groups.-GKOS also analyze how the impulse responses vary across income groups. They sort individuals based on their average earnings over the last years, and then compute the impulse responses within each group. Comparing the impulse responses across groups, they find that positive shock$\mathrm{s}$ are more persistent than negative shocks for low-earnings individuals. Since the opposite holds true for high-earnings individuals, putting together the impulse responses of different income groups yields what GKOS call a "butterfly pattern": As one moves up the distribution of recent earnings, the positive and negative branches of the impulse responses rotate in opposite directions.

Fig. 10 reports the results of a similar experiment on our simulated dataset. As in GKOS data, low-earnings individuals face larger shocks. Another similarity is that the negative shocks faced by high-earnings individuals are much larger than the positive shocks. But the simulations do not generate a butterfly pattern. We do not observe a significant correlation between recent earnings and the persistence of positive and negative shocks. Note, however, that the butterfly pattern might not be a universal feature of earnings data since ABB find that a different pattern holds true in PSID data. Using a semi-parametric approach, $\mathrm{ABB}$ show that persistence is at its strongest when high earnings households are hit by a good shock, or when low earnings households are hit by a bad shock.

\footnotetext{
${ }^{21}$ Today's earnings changes, $\log \left(c_{t}\right)-\log \left(c_{t-1}\right)$, go from -.55 to .5 in our simulation instead of -1.2 to 1 in GKOS data.
} 

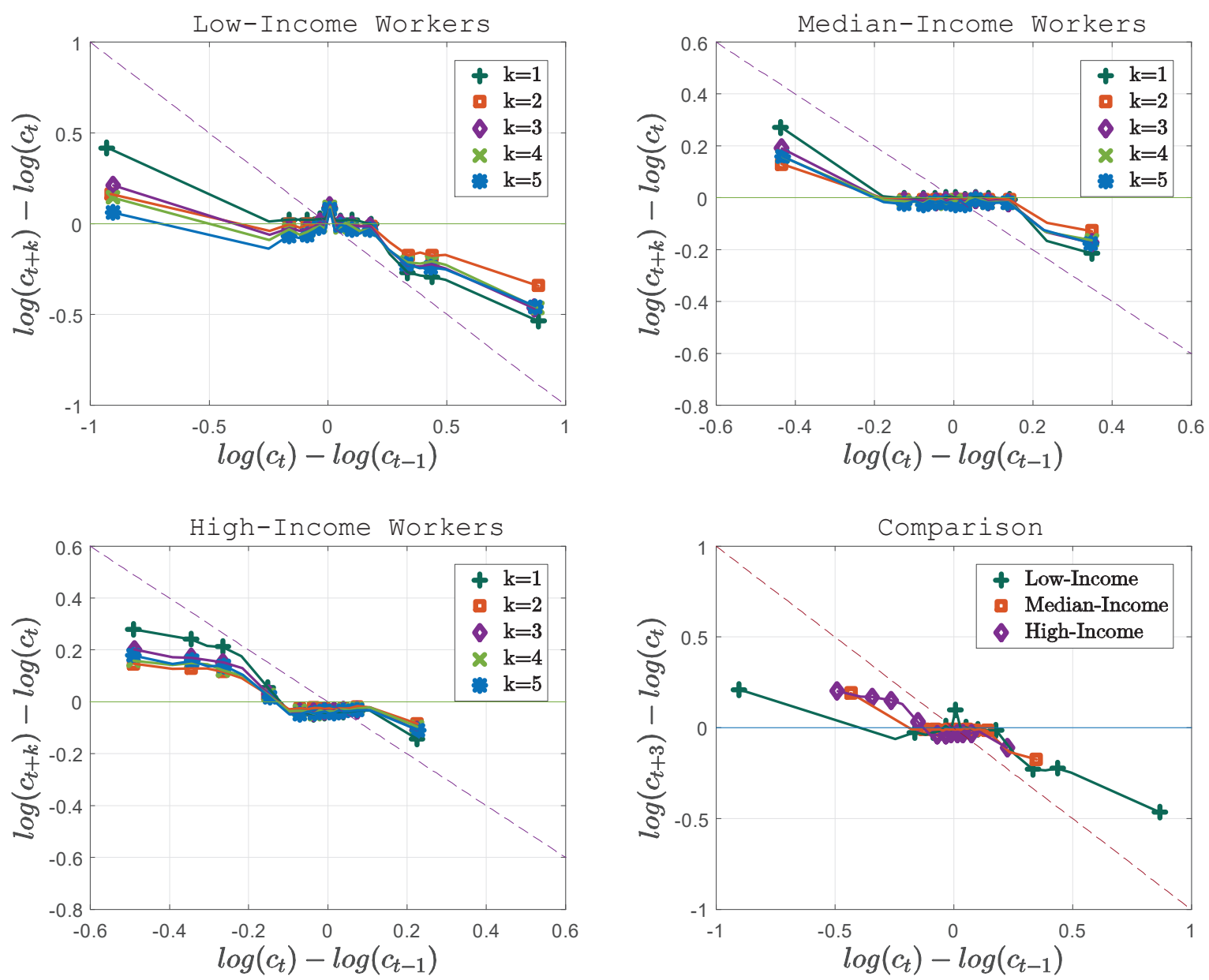

Figure 10: IMPUlSe ResPonses by INCOME GROUPS.

Notes: Parameters reported in Table 1. Low, median and high-income workers refer to workers whose income in $t-1$ is, respectively, below the second decile, between the fourth and sixth deciles, and above the eight decile. 


\section{Discussion}

\subsection{Related models}

The rest point of our model has the following two features:

(i) effort is below its output-maximizing level so that higher effort today raises current earnings and utility,

(ii) higher effort today does not raise future output.

Properties $(i)$ and $(i i)$ combine to generate the movements in the effective discoun$\mathrm{t}$ factor that are needed to generate cyclical effort paths. Models of human capital investment do not include both of these features.

Ben-Porath type of human capital investment.-Reputation, as we have modeled it, differs from traditional models of the Ben-Porath (1967) type in which a worker sacrifices current income in return for higher future earnings. In contrast to $(i)$ and (ii) such human capital investment lowers current earnings via a compensating differential contrary to property $(i)$, and it raises future earnings contrary to property (ii).

Learning by doing investment.-Although it does not have property (ii), LBD is the closest-related line of research because it potentially shares with our model property $(i)$, namely the feature that higher learning today raises an agent's income today. That does not mean, however, that it also raises today's utility. Here is a version of LBD that compares closely to our model. Let $k$ denote the stock of human capital and $a$ the investment in human capital. Assume that

$$
\begin{aligned}
& c_{t}=k_{t}+a_{t} \text { and, } \\
& k_{t}=(1-\delta) k_{t-1}+a_{t-1}, \text { with } \delta \in(0,1) .
\end{aligned}
$$

Property $(i)$ does not hold because agents set $a_{t}$ beyond the point where today's utility $U\left(c_{t}-g\left(a_{t}\right)\right)$ is maximized. ${ }^{22}$ Hence increasing $a_{t}$ reduces current utility instead of

\footnotetext{
${ }^{22}$ Current output is maximized when $a=g^{-1}(1)$. Thus the observation that effort exceeds its outputmaximizing level directly follows from the first-order condition$$
g^{\prime}\left(a_{t}\right)=1+\sum_{s=t+1}^{\infty} \beta^{s-t} \frac{U^{\prime}\left(c_{s}-g\left(a_{s}\right)\right)}{U^{\prime}\left(c_{t}-g\left(a_{t}\right)\right)} \frac{\partial k_{s}}{\partial a_{t}}>1
$$ 
raising it. Property (ii) does not hold either because, according to (26), higher effort raises $k^{\prime}$, and so increases tomorrow's output. Thus the discount factor is decreasing in $a_{t}$ at the rest point, and LBD exerts a stabilizing force which prevents the emergence of cycles.

When combined with technological choice, however, the LBD model can generate cycles resembling the asymmetric cycles displayed in Fig. 12 below. Examples are Parente (1994) and Jovanovic and Nyarko (1996).

\subsection{Other Extensions}

1. Non-stationary learning and the age-volatility relation.-We now describe four extensions that would extend further the model's explanatory power. As a first check, we let the precision of the starting prior $\sigma_{\theta, 0}^{-2}$ differ from its long-run level $\bar{\sigma}_{\theta}^{-2}$. We typically expect the precision of beliefs to increase over time, as is always the case when types are constant since then $\sigma_{\nu}^{-2}$, and thus $\bar{\sigma}_{\theta}^{-2}$, are both infinite. The return$s$ to effort are decreasing in posterior precision because it lowers the weight that the market puts on recent observations. Hence the equilibrium sequence of actions tend$s$ to decrease over time as in Holmström's model with risk-neutral agents. Adding risk-aversion allows us to construct another equilibrium sequence which oscillates around the regularly decreasing path, as shown in Fig. 11. This alternative solution eventually coincides with the two-period cycles described in the previous section because the precision converges to its stationary level $\bar{\sigma}_{\theta}^{-2}$. Moreover, the decreasing amplitude of the oscillations is consistent with empirical evidence about the negative relationship between income volatility and worker age.

Even along the regularly decreasing "Holmström" path shown by the solid curve in Fig. 11, income volatility is decreasing with the worker age. Cycling $a_{t}$ paths thus reinforce this decline by subtracting mass from the tails of the growth rates' distributions as the worker ages.

2. Asymmetric cycles and income-growth persistence.-Although we have focused on cycles of period 2, the model can generate more complex patterns. For example, it is possible to use the model with linear costs to generate cycles with more than two states. As an illustration, Fig. 12 describes incentive-compatible cycles of period 3 where investment grows for two successive periods and then drops back to its initial level. This is the simplest instance of asymmetric cycles featuring protracted 


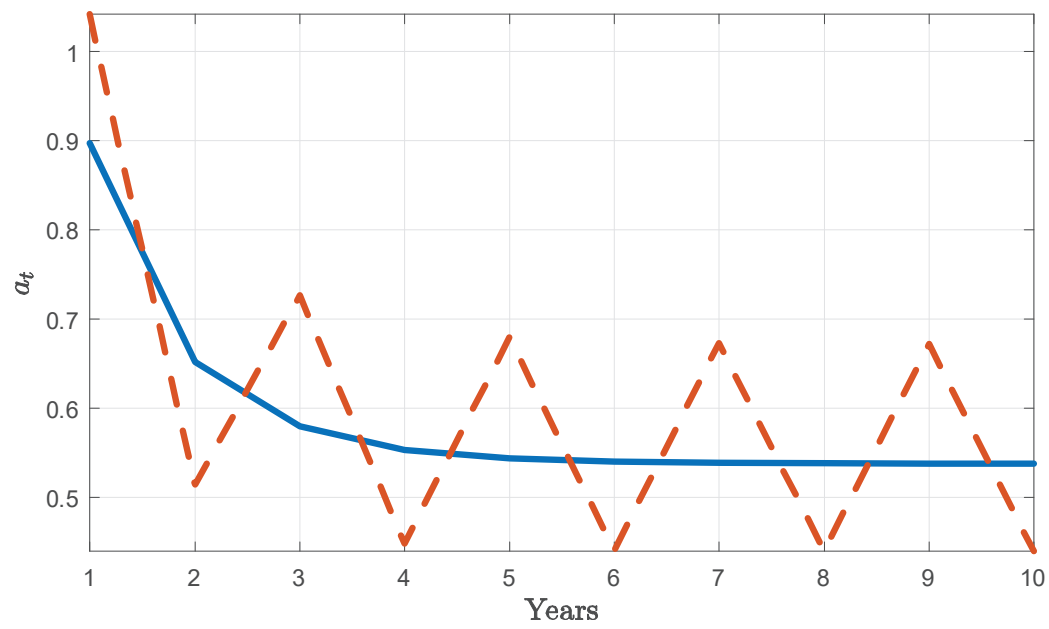

Figure 11: Even Wider TAils at Younger Ages Induced by CyCLing EFFort $a_{t}$.

booms and sudden busts. A tendency for a time series to show large negative growth rates followed by several smaller positive growth rates is known as "steep asymmetry." Moreover, the long-run frequency distribution of $a_{t}$ places equal weight on the points $0,0.71$, and 1 , and thus has longer left tail. A tendency for a detrended time series to have negative skew is known as "deep". ${ }^{23}$

The longer period of growth is sustainable because investment costs $\kappa$ are low, i.e., $\kappa<(1-\lambda) /\left(r^{-1}-\lambda\right) .{ }^{24}$ As explained in Section 3, when this inequality holds, the rest point is efficient because the agent always finds it profitable to raise her investment. But now she is discouraged from doing so in the two low states by the fact that the marginal utility of consumption will decrease in the following period. We show in Appendix A.3 that this feature gives rise to asymmetric cycles similar to the one depicted in Fig. 12 whenever: (i) $\bar{a}$ is high enough, and (ii) $\kappa \in\left(\underline{\kappa},(1-\lambda) /\left(r^{-1}-\lambda\right)\right)$ for some $\underline{\kappa}>0$. The logic of the proof can be extended to cycles with more than 3 states.

3. Finite horizon does not eliminate cycles.-In contrasting our model to Holmström's benchmark, we have stressed that equilibrium uniqueness is not anymore guaranteed. But one may argue that this distinction is not fundamental because it stems from our focus on Markov equilibria. Although this requirement is implicitly assumed in much, if not all, of the career concerns literature, there can be other solutions if one allows for strategies that are not Markov. However, whenever the agent

\footnotetext{
${ }^{23}$ See Fig. 4 in Jovanovic (2006) for a graphical distinction between the two types of asymmetry.

${ }^{24}$ By contrast, asymmetric cycles with protracted slumps and sudden booms can be constructed when the steady-state is inefficient, so that $\kappa>(1-\lambda) /\left(r^{-1}-\lambda\right)$.
} 


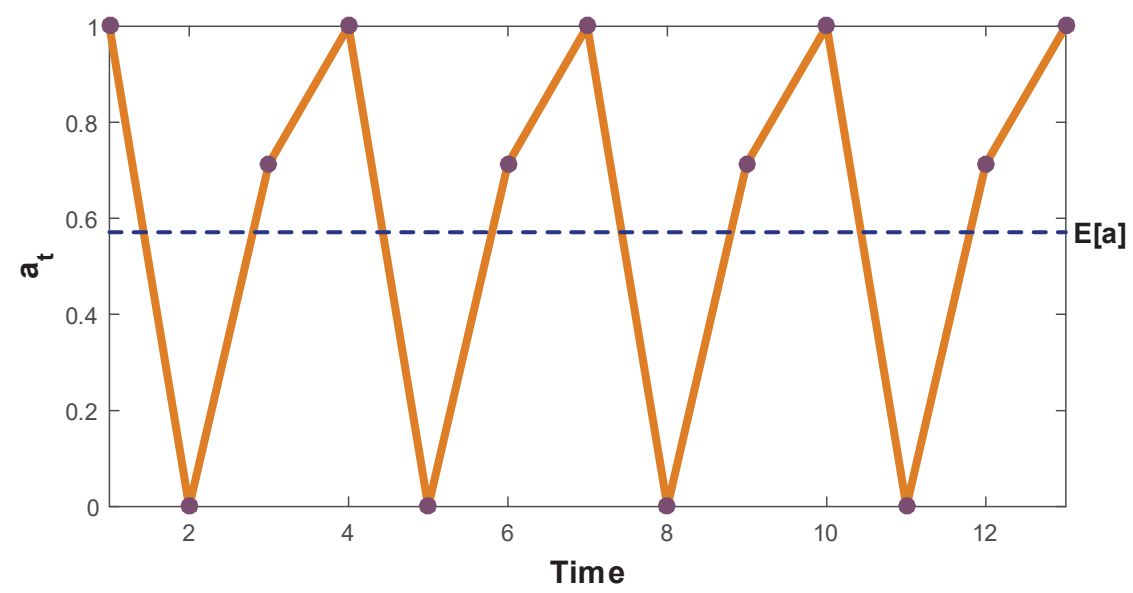

Figure 12: ASYMMETRIC CyCLES With CARA UTILITY AND LINEAR COSTS. Notes: $\sigma_{\varepsilon}=.1, \sigma_{\nu}=.2, \beta=.8, \gamma=4, \kappa=.75, \mathcal{A}=[0,1]$.

has a finite horizon, backward induction bites and the equilibrium under risk neutrality is unique and deterministic. By contrast, multiple equilibria and reputation cycles continue to arise in finite-horizon models with risk averse agents. ${ }^{25}$

4. General equilibrium extension.-The mechanisms described in this paper fits easily into a general equilibrium setting. Following the approach described in Jovanovic and Prat (2016), one recovers the same incentive structure if firms operate the technology described in (1) and if risk-averse households fully diversify their purchases of goods over firms. The only fundamental difference is that cycles are now in aggregate consumption. Hence the requirement that agents cannot smooth them becomes equivalent to the assumption that the economy is closed with no access to outside finance. Then the results on stability and cycles presented in Section 4 continue to hold in a macro setting. If anything, they hold more generally because the representative family runs many firms and is insured against firm-level shocks. The lack of idiosyncratic risk implies that deterministic equilibria are sustainable even when there is a wealth effect, thus making it possible to consider utility functions outside of the CARA family. In Jovanovic and Prat (2016) we show that a calibrated version of the macro model produces realistic fluctuations in terms of peak-to-trough

\footnotetext{
${ }^{25}$ This is particularly simple to check when disutility is linear. Let $T$ denote the agent's lifetime and assume that, as in Proposition $4, \kappa \in\left(\frac{1-\lambda}{r^{-1}-\lambda}, 1\right)$. Then $a_{t}^{*}=0$ for all $t \in\{1,2, \ldots, T\}$ is a deterministic equilibrium. But it is not always unique. For example, $a_{1}^{*}=\bar{a}$ and $a_{t}^{*}=0$ for all $t \in\{2, \ldots, T\}$ is also a deterministic equilibrium if the horizon $T$ and the upper bound $\bar{a}$ of the action set are large enough.
} 
movements in consumption and of the spacing of time between recessions.

\section{Conclusion}

We have shown that financial constraints can promote investment in reputation: Cyclical paths entail higher earnings because average effort is higher. This contrasts sharply with the findings reported in a host of papers that financial constraints inhibit tangible investment. It is likely, therefore, that when facing such constraints, firms may substitute away from investing in physical capital and towards reputation capital. The conditions under which this occurs are stated in Propositions 3 and 6, and the calibrated parameter values satisfy them comfortably.

We have also shown that cyclical effort paths generate unusual shocks to earnings and can help explain the evidence on their dynamics. By generating a large switch in effort, tail events in earnings growth emerge. The mechanism may not be the exact reputational mechanism that our model generates, but we have shown that the main alternative, learning by doing, is unlikely to be helpful in this regard. Instead one may hope to improve the model's fit further by embedding the reputation-concerns mechanism into a standard model of income dynamics. As documented in GKOS, such a richer model would include a mixture of autoregressive processes with different degrees of persistence, along with transitory shocks and abrupt transitions capturing the risk of non-employment.

The model also offers a new perspective on changes in the volatility of income, namely that a rise in income volatility could be the result of a rise in reputational concerns that, in turn could be called by a reduction in the noise accompanying the relation between effort and output. Agency theory suggests that a reduction in noise raises the incentives to provide effort, and we have shown that this can also help explain the rise in the volatility of earnings.

\section{References}

[1] Arellano, Manuel, Blundell, Richard and Stéphane Bonhomme. "Earnings and Consumption Dynamics: A Nonlinear Panel Data Framework." Econometrica, vol. 85(3) (2017): 693-734. 
[2] Bar-Isaac, Heski and Johannes Hörner. "Specialized Careers.” Journal of Economics and Management Strategy, vol. 23(3) (2014): 601-627.

[3] Ben-Porath, Yoram. "The Production of Human Capital and the Life Cycle of Earnings.” Journal of Political Economy, vol. 75(4) (1967): 352-365.

[4] Board, Simon and Moritz Meyer-ter-Vehn. "Reputation for Quality." Econometrica, vol. 81(6) (2013): 2381-2462.

[5] Cisternas, Gonzalo. "Two-Sided Learning and the Ratchet Principle." Review of Economic Studies, vol. 85(1) (2018): 307-351.

[6] Fazzari, Steven, Glenn Hubbard, and Bruce Petersen. "Financing Constraints and Corporate Investment." Brookings Papers on Economic Activity, vol. 19 (1988): 141-206.

[7] Green, Edward, and Robert Porter. "Noncooperative collusion under imperfect price information.” Econometrica, vol. 52(1) (1984): 87-100.

[8] Guvenen, Fatih, Fatih Karahan, Serdar Ozkan and Jae Song. "What Do Data on Millions of U.S. Workers Reveal about Life-Cycle Earnings Dynamics?” NBER Working Papers No. 20913 (2015).

[9] Holmström, Bengt. "Managerial Incentive Problems: A Dynamic Perspective." Review of Economic Studies, vol. 66 (1999): 169-182.

[10] Hubmer, Joachim. "The Job Ladder and its Implications for Earnings Risk." Review of Economic Dynamics, vol. 29 (2018): 172-194.

[11] Jovanovic, Boyan. "Asymmetric Cycles." The Review of Economic Studies, vol. 73(1) (2006): 145-162.

[12] Jovanovic, Boyan, and Yaw Nyarko. "Learning by doing and the choice of technology.” Econometrica, vol. 64(6) (1996): 1299-1310.

[13] Jovanovic, Boyan, and Julien Prat. “Reputation Cycles.” NBER Working Paper No. 22703 (2016).

[14] Kambourov, Gueorgui and Iourii Manovskii. "Rising Occupational and Industry Mobility in the United States: 1968-1997." International Economic Review, vol. 49(1) (2008): 41-79. 
[15] Kaplan, Greg, Giovanni L. Violante, and Justin Weidner. “The Wealthy Hand-tomouth.” Brookings Papers on Economic Activity, vol. 45(1) (2014): 77-153.

[16] Lange, Fabian. “The Speed of Employer Learning.” Journal of Labor Economics, vol. 25(1) (2007): 1-35.

[17] Mailath, George J., and Larry Samuelson. Repeated Games and Reputations. Oxford University Press, 2006.

[18] Parente, Stephen. "Technology Adoption, Learning-by-Doing, and Economic Growth.” Journal of Economic Theory, vol. 63(2) (1994): 346-369.

[19] Shimer, Rob. "Convergence in Macroeconomics: The Labor Wedge." American Economic Journal: Macroeconomics, vol. 1(1) (2009): 280-97.

[20] Song, Jae, David Price, Fatih Guvenen, Nicholas Bloom, and Till von Wachter. "Firming Up Inequality." NBER Working Papers No. 21199 (2015). 


\section{A Appendix}

\section{A.1 Proof of Proposition 1}

We prove that, given the unique consumption function that satisfies the no-profit requirement, the optimality of a conjectured deterministic profile can be established by a first-order condition. In Holmström (1999) sufficiency of the necessary condition immediately followed from the linear impact of actions on payoffs. With concave payoffs, we have to characterize the agent's problem both on and off the equilibrium path. Hence we consider arbitrary strategies and let $\delta_{t} \equiv a_{t}-a_{t}^{*}$ denote the deviation from conjectured effort at each date. We wish to relate the agent's and market's posteriors about $\theta$, which we denote $m^{a}$ and $m$, respectively. Bayes rule implies that

$$
m_{t+1}^{a}-m_{t+1}=\frac{1-\lambda}{\lambda} \sum_{s=0}^{t} \lambda^{t-s}\left(x_{s}^{a}-x_{s}\right)=-\frac{1-\lambda}{\lambda} \sum_{s=0}^{t} \lambda^{t-s} \delta_{s}
$$

where $x_{t}^{a} \equiv y_{t}-a_{t}$. Let $\Delta_{t}$ denote the following weighted mean of past deviations

$$
\Delta_{t}=\lambda \Delta_{t-1}+(1-\lambda) \delta_{t-1} \text { with } \Delta_{0}=0 .
$$

It follows from (28) that $m_{t}^{a}=m_{t}-\Delta_{t}$. We can use this relationship to specify the agent's expectations about the law of motion of beliefs. First notice that $y_{t}-m_{t}^{a}=$ $\theta_{t}-m_{t}^{a}+a_{t}+\varepsilon_{t}$. Since $\theta_{t}-m_{t}^{a}$ is normally distributed with mean 0 and variance $\bar{\sigma}_{\theta}^{2}$, the innovation process from the agent's standpoint is a normally distributed variable, $u \sim \mathcal{N}\left(0, \bar{\sigma}_{\theta}^{2}+\sigma_{\varepsilon}^{2}\right)$, such that $y_{t}=m_{t}^{a}+a_{t}+u_{t}$. Reinserting this decomposition into the law of motion of market's beliefs, we find that

$$
m_{t+1}=\lambda m_{t}+(1-\lambda) x_{t}=m_{t}+(1-\lambda)\left[\delta_{t}-\Delta_{t}+u_{t}\right] .
$$

The normality of $u_{t}$ implies that the distribution of the posterior $P\left(m_{t+1}\right)$ satisfies

$$
P\left(m_{t+1} \mid m_{t}, \Delta_{t}, \delta_{t}\right)=\Phi\left(\frac{m_{t+1}-\left[m_{t}+(1-\lambda)\left(\delta_{t}-\Delta_{t}\right)\right]}{(1-\lambda)\left(\bar{\sigma}_{\theta}+\sigma_{\varepsilon}\right)}\right)
$$

where $\Phi(\cdot)$ is the standard normal CDF. Given the per-period utility function $U\left(m, a^{*}, \delta\right)=$ $-\exp \left(-\gamma\left[m+a^{*}-g\left(a^{*}+\delta\right)\right]\right)$, the agent's value function on and off the equilibrium 
path is given by the fixed point of the following functional equation

$$
\begin{aligned}
V_{t}(m, \Delta) & =\max _{\delta}\left\{U\left(m, a_{t}^{*}, \delta\right)+\beta \int V_{t+1}\left(m^{\prime}, \Delta^{\prime}\right) d P\left(m^{\prime} \mid m, \Delta, \delta\right)\right\}, \\
\text { s.t. } m^{\prime} & =m+(1-\lambda)[\delta-\Delta+u], \\
\Delta^{\prime} & =\lambda \Delta+(1-\lambda) \delta .
\end{aligned}
$$

Lemma 1 Assume that the per-period utility function is CARA:

$$
\mathbf{U}(c, a)=U(c-g(a))=-\exp (-\gamma(c-g(a))), \text { with } \gamma>0 .
$$

Given a deterministic profile $\left\{a_{t}^{*}\right\}_{t=0}^{\infty}$, optimal strategies on and off the equilibrium path are deterministic functions of time and of the stock of past deviations $\Delta_{t}=\lambda \Delta_{t-1}+$ $(1-\lambda)\left(a_{t}-a_{t}^{*}\right)$, where $\Delta_{0}=0$. In particular, they do not depend on the agent's reputation $m_{t}$ nor on her output history.

Proof. The lemma follows from the fact that the value function is of the form

$$
V_{t}(m, \Delta)=\exp (-\gamma m) V_{t}(0, \Delta) .
$$

To verify the conjecture, we change the variable of integration of the Bellman equation

$$
\begin{aligned}
V_{t}(m, \Delta) & =\max _{\delta}\left\{\begin{array}{l}
-\exp \left(-\gamma\left[m+a_{t}^{*}-g\left(a_{t}^{*}+\delta\right)\right]\right) \\
+\beta \int V_{t+1}\left(m+(1-\lambda)[\delta-\Delta+u], \Delta^{\prime}\right) d \Phi\left(\frac{u}{\overline{\sigma_{\theta}}+\sigma_{\epsilon}}\right)
\end{array}\right\} \\
& =\exp (-\gamma m) \max _{\delta}\left\{\begin{array}{l}
-\exp \left(-\gamma\left[a_{t}^{*}-g\left(a_{t}^{*}+\delta\right)\right]\right) \\
+\beta \int V_{t+1}\left((1-\lambda)[\delta-\Delta+u], \Delta^{\prime}\right) d \Phi\left(\frac{u}{\bar{\sigma}_{\theta}+\sigma_{\epsilon}}\right)
\end{array}\right\} \\
& =\exp (-\gamma m) V_{t}(0, \Delta) .
\end{aligned}
$$

The second equality follows reinserting our guess (32) and using the fact that $a_{t}^{*}$ is deterministic and thus independent of $m$, while the third equality follows directly from the Bellman equation (30) defining $V$. Since the functional equation is a contraction mapping, the value function is unique and its policy function $\delta(\Delta, t)$ identical across all market's beliefs $m$. Hence, the agent chooses a sequence of deterministic actions that only depend on the cumulative stock of past deviations $\Delta$ and potentially on $t$. In other words, optimal policies are deterministic on and off the equilibrium path. 
We are now in a position to prove Proposition 1. According to Lemma 1, there is no loss of generality in focusing on sequences of deterministic actions. Let $\mathbf{a} \equiv$ $\left\{a_{0}, a_{1}, \ldots\right\}$ denote a sequence of actions from date 0 onwards. Given that effort in each period belongs to the feasibility set $\mathcal{A} \subseteq \mathbb{R}_{+}$, sequences are element of the infinite Cartesian product $\mathcal{A}^{\infty}=\left\{\left(a_{t}\right)_{t=0}^{\infty} \mid a_{t} \in \mathcal{A}\right.$ for all $\left.t=0,1, \ldots\right\}$. The market belief can be computed using the Bayesian map

$$
m_{t+1}\left(\mathbf{a}, \mathbf{x}^{a} \mid m_{0}\right)=\lambda^{t} m_{0}+\frac{1-\lambda}{\lambda} \sum_{s=0}^{t} \lambda^{t-s}\left(x_{s}^{a}-a_{s}^{*}+a_{s}\right),
$$

where $\mathbf{x}^{a} \equiv\left\{x_{t}\right\}_{t=0}^{\infty}$. We use these notations to define the agent's utility as a functional in the space of action sequences

$$
V_{0}(0,0)=\max _{\mathbf{a} \in \mathcal{A}^{\infty}} \mathcal{U}(\mathbf{a})=\max _{\mathbf{a} \in \mathcal{A}^{\infty}} E\left[-\sum_{t=0}^{\infty} \beta^{t} \exp \left(-\gamma\left[m_{t}(\mathbf{a})+a_{t}^{*}-g\left(a_{t}\right)\right]\right)\right],
$$

where $m_{t}(\mathbf{a})$ is a shorthand version of $m_{t}\left(\mathbf{a}, \mathbf{x}^{a} \mid m_{0}\right)$ introduced in (33). ${ }^{26}$ Sufficiency of the necessary condition will follow if we can prove that $\mathcal{U}(a)$ is a concave functional. We establish an even stronger requirement as concavity holds in all periods. To see why, let $p_{t}(\mathbf{a}) \equiv c_{t}(\mathbf{a})-g\left(a_{t}\right)$ denote returns in period $t$, i.e., consumption net of the disutility of effort. We have

$$
\begin{aligned}
p_{t}\left(\alpha \mathbf{a}^{1}+(1-\alpha) \mathbf{a}^{2}\right) & =m_{t}\left(\alpha \mathbf{a}^{1}+(1-\alpha) \mathbf{a}^{2}\right)+a_{t}^{*}-g\left(\alpha a_{t}^{1}+(1-\alpha) a_{t}^{2}\right) \\
& \geq \alpha\left[m_{t}\left(\mathbf{a}^{1}\right)+a_{t}^{*}-g\left(a_{t}^{1}\right)\right]+(1-\alpha)\left[m_{t}\left(\mathbf{a}^{2}\right)+a_{t}^{*}-g\left(a_{t}^{2}\right)\right] \\
& =\alpha p_{t}\left(\mathbf{a}^{1}\right)+(1-\alpha) p_{t}\left(\mathbf{a}^{2}\right),
\end{aligned}
$$

for any $\alpha \in(0,1)$ and all pair of sequences $\mathbf{a}^{1}, \mathbf{a}^{2} \in \mathcal{A}^{\infty}$. The first equality uses the fact that the market conjecture $a_{t}^{*}$ is deterministic. The second inequality holds true because the disutility $g$ is convex in $a$, while the Bayesian map for $m_{t}$ is linear in a, so that $m_{t}\left(\alpha \mathbf{a}^{1}+(1-\alpha) \mathbf{a}^{2}\right)=\alpha m_{t}\left(\mathbf{a}^{1}\right)+(1-\alpha) m_{t}\left(\mathbf{a}^{2}\right)$. Per-period utility is given by $U_{t}(\mathbf{a})=\left(U \circ p_{t}\right)(\mathbf{a})$, and since the composition of concave continuous functions is itself concave, the strict concavity of $U$ along with the convexity of $\mathcal{A}$ imply that

$$
U_{t}\left(\alpha \mathbf{a}^{1}+(1-\alpha) \mathbf{a}^{2}\right)>\alpha U_{t}\left(\mathbf{a}^{1}\right)+(1-\alpha) U_{t}\left(\mathbf{a}^{2}\right),
$$

\footnotetext{
${ }^{26}$ Since the innovation process $\mathbf{x}^{a}$ and the prior $m_{0}$ cannot be influenced by the agent, they can be treated as exogenous when studying her optimal control problem.
} 
for any $\alpha \in(0,1)$ and all pair of sequences $\mathbf{a}^{1} \neq \mathbf{a}^{2} \in \mathcal{A}^{\infty}$. We have therefore proven that per-period utility is strictly concave in a, which ensures that

$$
\begin{aligned}
\mathcal{U}\left(\alpha \mathbf{a}^{1}+(1-\alpha) \mathbf{a}^{2}\right) & =E\left[\sum_{t=0}^{\infty} \beta^{t} U_{t}\left(\alpha \mathbf{a}^{1}+(1-\alpha) \mathbf{a}^{2}\right)\right] \\
& >\alpha E\left[\sum_{t=0}^{\infty} \beta^{t} U_{t}\left(\mathbf{a}^{1}\right)\right]+(1-\alpha) E\left[\sum_{t=0}^{\infty} \beta^{t} U_{t}\left(\mathbf{a}^{2}\right)\right] \\
& =\alpha \mathcal{U}\left(\mathbf{a}^{1}\right)+(1-\alpha) \mathcal{U}\left(\mathbf{a}^{2}\right) .
\end{aligned}
$$

Note that the inequality holds true because the only source of randomness is the innovation process $\mathbf{x}^{a}$ defined from the agent's standpoint so that its realizations are by definition independent of $\mathbf{a}$. We can therefore conclude that $\mathcal{U}$ is a strictly concave functional in the space of action sequences. Thus any local maximum of $\mathcal{U}$ is also a global maximum. A necessary condition for $\mathcal{U}$ to have a maximum at $\mathbf{a}^{*}$ is that its Gateaux derivative $d \mathcal{U}\left(\mathbf{a}^{*} ; h\right)=0$ for all $h \in \mathcal{A}^{\infty}$. The existence of the Gateaux derivative being guaranteed by the convexity of $\mathcal{A}^{\infty}$, this requirement yields the necessary FOC (11) which, by strict concavity of the objective, is also sufficient.

\section{A.2 Proofs of Propositions 2 to 7}

Proof. Proposition 2: We wish to show that the necessary conditions (10) and (11) are equivalent when the agent's utility is CARA. Reinserting (9) into (10), we find that (10) is satisfied when

$$
\begin{aligned}
g^{\prime}\left(a_{t}^{*}\right) & =\frac{1-\lambda}{\lambda} \sum_{s=t+1}^{\infty}(\beta \lambda)^{s-t} E_{t}^{*}\left[\frac{U^{\prime}\left(c_{s}-g\left(a_{s}^{*}\right)\right)}{U^{\prime}\left(c_{t}-g\left(a_{t}^{*}\right)\right)}\right] \\
& =\frac{1-\lambda}{\lambda} \sum_{s=t+1}^{\infty}(\beta \lambda)^{s-t} E_{t}^{*}\left[\exp \gamma\left(m_{t}+a_{t}^{*}-g\left(a_{t}^{*}\right)-\left(m_{s}+a_{s}^{*}-g\left(a_{s}^{*}\right)\right)\right)\right] .
\end{aligned}
$$

The posterior $m$ cancels out from the expectation because

$$
m_{t+1}=\lambda m_{t}+(1-\lambda) x_{t}=m_{t}+(1-\lambda)\left(\theta_{t}-m_{t}+\varepsilon_{t}\right),
$$


so that

$$
\begin{aligned}
E_{t}^{*}\left[\exp \left(\gamma\left(m_{t}-m_{s}\right)\right)\right] & =E_{t}^{*}\left[\exp \left(-\gamma(1-\lambda) \sum_{i=0}^{s-1}\left(\theta_{t+i}-m_{t+i}+\varepsilon_{t+i}\right)\right)\right] \\
& =\exp \left(\frac{\gamma^{2}(1-\lambda)^{2}\left(\sigma_{\varepsilon}^{2}+\bar{\sigma}_{\theta}^{2}\right)(s-t)}{2}\right) .
\end{aligned}
$$

The second equality holds true because (i) $\theta_{t} \sim \mathcal{N}\left(m_{t}, \bar{\sigma}_{\theta}^{2}\right)$, and (ii) $\varepsilon_{t}$ is a normally distributed noise with variance $\sigma_{\varepsilon}^{2}$. The expression above can be simplified using the definition of $\bar{\sigma}_{\theta}^{2}$ in (6) and the fact that $\lambda=\bar{\sigma}_{\theta}^{2} /\left(\sigma_{\varepsilon}^{2}+\bar{\sigma}_{\theta}^{2}\right)$, hence

$$
\begin{aligned}
(1-\lambda)^{2}\left(\sigma_{\varepsilon}^{2}+\bar{\sigma}_{\theta}^{2}\right) & =\frac{\bar{\sigma}_{\theta}^{4}}{\sigma_{\varepsilon}^{2}+\bar{\sigma}_{\theta}^{2}}=\frac{4 \sigma_{\varepsilon}^{4}\left(\sqrt{1+\frac{4 \sigma_{\varepsilon}^{2}}{\sigma_{\nu}^{2}}}-1\right)^{-2}}{\sigma_{\varepsilon}^{2}\left[1+2\left(\sqrt{1+\frac{4 \sigma_{\varepsilon}^{2}}{\sigma_{\nu}^{2}}}-1\right)^{-1}\right]} \\
& =\frac{4 \sigma_{\varepsilon}^{2}}{\left(\sqrt{1+\frac{4 \sigma_{\varepsilon}^{2}}{\sigma_{\nu}^{2}}}-1\right)\left(\sqrt{1+\frac{4 \sigma_{\varepsilon}^{2}}{\sigma_{\nu}^{2}}}+1\right)}=\sigma_{\nu}^{2} .
\end{aligned}
$$

Simplifying the expectation in (35) and replacing the resulting expression into (34), we finally obtain

$$
\begin{aligned}
g^{\prime}\left(a_{t}^{*}\right) & =\frac{1-\lambda}{\lambda} \sum_{s=t+1}^{\infty}(\beta \lambda)^{s-t} E_{t}^{*}\left[\frac{U^{\prime}\left(c_{s}-g\left(a_{s}^{*}\right)\right)}{U^{\prime}\left(c_{t}-g\left(a_{t}^{*}\right)\right)}\right] \\
& =\frac{1-\lambda}{\lambda} \sum_{s=t+1}^{\infty}(\beta \lambda)^{s-t} \exp \left(\frac{\gamma^{2} \sigma_{\nu}^{2}(s-t)}{2}\right) \exp \left(\gamma\left[a_{t}^{*}-g\left(a_{t}^{*}\right)-\left(a_{s}^{*}-g\left(a_{s}^{*}\right)\right)\right]\right),
\end{aligned}
$$

which is indeed equivalent to (11) since $\beta^{s-t} \exp \left(\gamma^{2} \sigma_{\nu}^{2}(s-t) / 2\right)=r^{s-t}$.

Proof. Propositions 3 and 4: Since Proposition 3 is a subcase of Proposition 4, we directly prove the general case where output is noisy, so that $\sigma_{\varepsilon}>0$. We are interested in 2-period cycles where $a_{t}^{*}=\bar{a}$ when $t$ is even, and $a_{t}^{*}=0$ when $t$ is odd. Using the notation

$$
S(a) \equiv \exp (\gamma(1-\kappa) a),
$$

and taking into account the feasibility constraint $a \in[0, \bar{a}]$, we see that the Kuhn- 
Tucker conditions resulting from the incentive compatibility constraint (10) are

$$
\kappa \leq \frac{1-\lambda}{\lambda}\left(\sum_{i \in\{1,3,5, \ldots\}}^{\infty}(r \lambda)^{i} S(\bar{a})+\sum_{i \in\{2,4,6, \ldots\}}^{\infty}(r \lambda)^{i}\right) \text { if } t \text { is even so that } a_{t}^{*}=\bar{a},
$$

and

$$
\kappa \geq \frac{1-\lambda}{\lambda}\left(\sum_{i \in\{1,3,5, \ldots\}}^{\infty}(r \lambda)^{i} S(\bar{a})^{-1}+\sum_{i \in\{2,4,6, \ldots\}}^{\infty}(r \lambda)^{i}\right) \text { if } t \text { is odd so that } a_{t}^{*}=0 .
$$

These two conditions can be re-written as

$$
\frac{1}{S(\bar{a})} \leq f(\kappa, r, \lambda) \leq S(\bar{a}), \text { where } f(\kappa, r, \lambda) \equiv \kappa\left[\frac{1-(r \lambda)^{2}}{r(1-\lambda)}\right]-r \lambda .
$$

The following cases have to be distinguished:

1. $\kappa \leq \frac{r^{2} \lambda-(r \lambda)^{2}}{1-(r \lambda)^{2}}$ : Then $f(\kappa, r, \lambda) \leq 0$ and, since $S(\bar{a})>0$, the first inequality in (39) cannot be satisfied. Intuitively, the costs $\kappa$ are so low that it is never optimal to set $a^{*}=0$.

2. $\kappa \in\left(\frac{r^{2} \lambda-(r \lambda)^{2}}{1-(r \lambda)^{2}}, \frac{r(1-\lambda)}{1-r \lambda}\right]$ : Then $f(\kappa, r, \lambda) \in(0,1]$ and, using the definition of $S(a)$ in (36), we find that the first inequality in (39), $1 / S(\bar{a}) \leq f(\kappa, r, \lambda)$, is satisfied whenever $\bar{a} \geq-\log (f(\kappa, r, \lambda)) /[\gamma(1-\kappa)]$. As for the second inequality, it follows from $1 / S(\bar{a}) \leq 1$ that $S(\bar{a}) \geq 1 \geq f(\kappa, r, \lambda)$.

3. $\kappa \in\left(\frac{r(1-\lambda)}{1-r \lambda}, 1\right)$ : Then $f(\kappa, r, \lambda)>1$ and the second inequality in (39), $f(\kappa, r, \lambda) \leq$ $S(\bar{a})$, is satisfied whenever $\bar{a} \geq \log (f(\kappa, r, \lambda)) /[\gamma(1-\kappa)]$. The first inequality immediately follows from $S(\bar{a}) \geq f(\kappa, r, \lambda)>1>1 / S(\bar{a})$.

Propositions 3 and 4 focus on case 3 . Since $f(\kappa, r, \lambda)$ is strictly decreasing in $r$, and $\lim _{r \rightarrow 0} f(\kappa, r, \lambda)=+\infty$ while $f\left(\kappa, \frac{\kappa}{1-\lambda+\lambda \kappa}, \lambda\right)=1$, there exists an $\underline{r}$ such that $S(\bar{a})>f(\kappa, r, \lambda)$ for all $r \in\left(\underline{r}, \frac{\kappa}{1-\lambda+\lambda \kappa}\right)$. Given that $S(\bar{a})$ is increasing in $\gamma$ and $\bar{a}$ and $\partial f(\kappa, \underline{r}, \lambda) / \partial r<0, \underline{r}$ must decrease when $\gamma$ or $\bar{a}$ increase in order to restore the equality $f(\kappa, \underline{r}, \lambda)=S(\bar{a})$. Note that when $\sigma_{\varepsilon}=0$, as in Proposition $3, \lambda$ is also equal to 0 and $f(\kappa, r, 0)=\kappa / r$. Then the condition $f(\kappa, r, 0) \leq S(\bar{a})$ is necessarily satisfied whenever $r \in(\kappa \exp (-\gamma(1-\kappa) \bar{a}), \kappa)$, as stated in Proposition 3. 


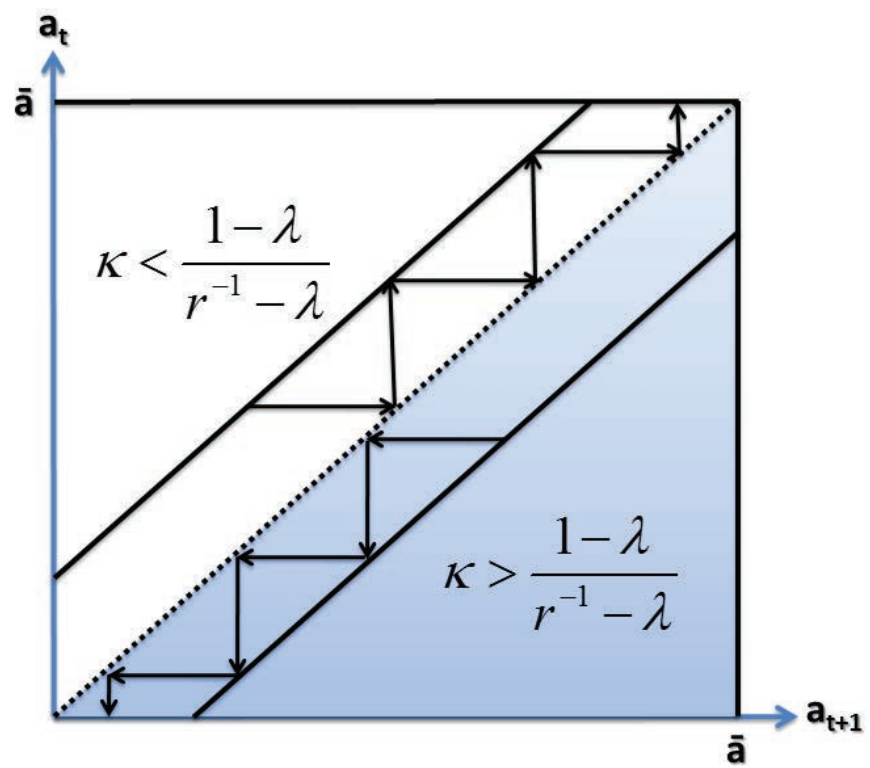

Figure 13: Phase Portraits For two Different VAlues of $\kappa$ WHEN $g(a)=\kappa a$.

Finally, we need to show that $\hat{a}=0$ is the unique rest point when $\kappa>(1-\lambda) /\left(r^{-1}-\right.$ $\lambda)$. Set $a_{s}^{*}=\hat{a}$ for all $s \geq t$ in (11). The resulting incentive constraint reads $\kappa=$ $r(1-\lambda+\lambda \kappa)$, a requirement that cannot be satisfied since we are focusing on cases where $\kappa>(1-\lambda) /\left(r^{-1}-\lambda\right)$. Hence $\hat{a}>0$ cannot be incentive compatible as the agent would like to deviate by investing less than $\hat{a}$, as shown in Fig. 13. However, such deviations are not feasible when $a_{t}^{*}=0$ for all $t$, and so $\hat{a}=0$ is indeed the only rest point.

Proof. Proposition 5: We first prove that cycles can emerge solely if $\sigma_{\varepsilon}$ does not exceed a certain threshold. The first step consists in rewriting the necessary condition (37) as follows

$$
\kappa \leq \frac{1-\lambda}{\lambda}\left(\sum_{i \in\{1,3,5, \ldots\}}^{\infty}(r \lambda)^{i} S(\bar{a})+\sum_{i \in\{2,4,6, \ldots\}}^{\infty}(r \lambda)^{i}\right)=\frac{r(1-\lambda)}{1-r \lambda}\left(\frac{S(\bar{a})-1}{1+r \lambda}+1\right) .
$$

The expression on the right-hand side is decreasing in $\lambda$ and converges to 0 as $\lambda$ goes to 1 . Hence there exists a $\bar{\lambda} \in(0,1)$ such that the necessary condition (37) is not satisfied whenever $\lambda>\bar{\lambda}$. Given that $\lambda$ is strictly increasing in $\sigma_{\varepsilon}$, and that it goes 
from 0 to 1 as $\sigma_{\varepsilon}$ goes from 0 to infinity, ${ }^{27}$ two-period cycles cannot be sustainable whenever $\sigma_{\varepsilon}$ is greater than a certain value, which we denote by $\bar{\sigma}_{\varepsilon}$.

(i) Let's now first consider cases where $\kappa \leq r$. If $\lambda=(r-\kappa) /[r(1-\kappa)]$, the definition of $f$ in (39) implies that $f(\kappa, r, \lambda)=1$. Given that $S(\bar{a})$ is greater than 1 , condition (39) is necessarily satisfied and so $\bar{\lambda}>(r-\kappa) /[r(1-\kappa)]$. Moreover, it follows from the continuity of $f$ and the proof of Proposition 4 that there exists a $\underline{\lambda} \leq(r-\kappa) /[r(1-\kappa)]$ such that the necessary condition is sufficient and satisfied for all $\lambda \in[\underline{\lambda}, \bar{\lambda}]$. Given the relationship between $\sigma_{\varepsilon}$ and $\lambda$, $\underline{\lambda}$ defines a unique $\underline{\sigma}_{\varepsilon}$ such that two-period cycles are sustainable for all $\sigma_{\varepsilon} \in\left[\underline{\sigma}_{\varepsilon}, \bar{\sigma}_{\varepsilon}\right]$.

(ii) We now consider cases where $\kappa>r$. The necessary condition is satisfied when $\lambda=0$ if and only if $f(\kappa, r, 0) \leq S(\bar{a})$, i.e., when $\bar{a} \geq \log (\kappa / r) /[\gamma(1-\kappa)]$. Then we also know from the first step of the proof that there exists a $\bar{\lambda}$ such that the necessary condition will be satisfied for all $\lambda \in[0, \lambda]$. Moreover, we have shown in Step 3 of the proof of Proposition 4 that the necessary condition is also sufficient whenever the rest point is inefficient, a property that is verified because $\kappa>r$. Finally, using once again the relationship between $\sigma_{\varepsilon}$ and $\lambda$, we conclude that two-period cycles are sustainable if and only if $\sigma_{\varepsilon} \in\left[0, \bar{\sigma}_{\varepsilon}\right]$.

Proof. Proposition 6: We want to characterize deterministic dynamics near the rest point $\hat{a}$. Let $\varphi\left(a_{t}\right)=a_{t+1}$ denote the implicit map so that $l(a)=h(\varphi(a))$, where $l(a) \equiv g^{\prime}(a) U^{\prime}(a-g(a))$ and $h(a) \equiv r U^{\prime}(a-g(a))\left[1-\lambda+\lambda g^{\prime}(a)\right]$. Differentiating the incentive constraint (18) at the rest point, we find that

$$
\left.\varphi^{\prime}(\hat{a}) \equiv \frac{d a_{t+1}}{d a_{t}}\right|_{a_{t}=\hat{a}}=\frac{A_{g}(\hat{a})-A_{U}(\hat{a}-g(\hat{a}))\left[1-g^{\prime}(a)\right]}{\frac{\lambda g^{\prime}(\hat{a})}{1-\lambda+\lambda g^{\prime}(\hat{a})} A_{g}(\hat{a})-A_{U}(\hat{a}-g(\hat{a}))\left[1-g^{\prime}(a)\right]},
$$

where $A_{U}(x)=-U^{\prime \prime}(x) / U^{\prime}(x)$ and $A_{g}(a)=g^{\prime \prime}(a) / g^{\prime}(a)$.

The rest point $\hat{a}$ is locally stable if $\left|\varphi^{\prime}(\hat{a})\right| \in[0,1)$. Since the numerator in (40) is always higher than the denominator, the stability condition can be satisfied solely if the denominator is negative. Let us focus first on cases where the numerator is positive while the denominator is negative. Then it is easy to verify that $\varphi^{\prime}(\hat{a}) \in(-1,0]$ whenever (20) is satisfied. The other possibility is that both numerator and denominator

\footnotetext{
${ }^{27}$ Remember that $\lambda=1-\frac{\sigma_{\varepsilon}^{-2}}{\bar{\sigma}_{\theta}^{-2}+\sigma_{\varepsilon}^{-2}}=1-2\left(\sqrt{1+4 \frac{\sigma_{\varepsilon}^{2}}{\sigma_{\nu}^{2}}}+1\right)^{-1}$.
} 
are negative, then

$$
\varphi^{\prime}(\hat{a}) \in[0,1) \Leftrightarrow A_{g}(\hat{a})<A_{U}(\hat{a}-g(\hat{a}))\left(1-g^{\prime}(\hat{a})\right),
$$

a condition that is always fulfilled when requirement (20) in Proposition 6 is satisfied.

Proposition 7 can be proved in a similar way to Proposition 4 . Thus we first use a direct approach by changing variable and defining a new fixed point problem.

\section{Lemma 2 Let}

$$
a(s) \equiv \frac{r(1-\lambda)}{1-(r \lambda)^{2}}(r \lambda+s),
$$

2-period cycles are sustainable when the fixed point problem

$$
s=\psi(s) \equiv \frac{U^{\prime}(c(a(1 / s))-g(a(1 / s)))}{U^{\prime}(c(a(s))-g(a(s)))}
$$

admits a solution $s^{*} \neq 1$.

Proof. Lemma 2: We focus on 2-period cycles and denote this period action by $a$, next period by $a^{\prime}$, the period after by $a$, and so on. In other words, we have the discount factors from today til tomorrow and from tomorrow til the day after, respectively,

$$
\begin{aligned}
s & \equiv U^{\prime}\left(c\left(a^{\prime}\right)-g\left(a^{\prime}\right)\right) / U^{\prime}(c(a)-g(a)), \\
s^{\prime} & \equiv 1 / s\left(a, a^{\prime}\right) .
\end{aligned}
$$

Therefore, if we start at $t=0$, so that $a$ is the action at $t=0,2,4,6 \ldots$ and $a^{\prime}$ the action for $t=1,3,5,7, \ldots$, then the incentive constraint (10) is satisfied when $\left(a, a^{\prime}\right)$ solve the following two equations

$$
\begin{aligned}
& a=\frac{1-\lambda}{\lambda}\left(\sum_{\mathrm{t}=1,3,5,7, \ldots}^{\infty}(r \lambda)^{t} s+\sum_{\mathrm{t}=2,4,6,8, \ldots}^{\infty}(r \lambda)^{t}\right)=\frac{1-\lambda}{\lambda}\left(\frac{r \lambda s}{1-(r \lambda)^{2}}+\frac{(r \lambda)^{2}}{1-(r \lambda)^{2}}\right) \\
& a^{\prime}=\frac{1-\lambda}{\lambda}\left(\sum_{\mathrm{t}=1,3,5,7, \ldots}^{\infty}(r \lambda)^{t} s^{\prime}+\sum_{\mathrm{t}=2,4,6,8, \ldots}^{\infty}(r \lambda)^{t}\right)=\frac{1-\lambda}{\lambda}\left(\frac{r \lambda s^{\prime}}{1-(r \lambda)^{2}}+\frac{(r \lambda)^{2}}{1-(r \lambda)^{2}}\right)
\end{aligned}
$$


These simplify to

$$
\begin{aligned}
a & =\frac{1-\lambda}{1-(r \lambda)^{2}} r(r \lambda+s), \\
a^{\prime} & =\frac{1-\lambda}{1-(r \lambda)^{2}} r\left(r \lambda+s^{\prime}\right) .
\end{aligned}
$$

Thus there are 4 equations (43), (44), (45), and (46), and 4 unknowns, $\left(a, a^{\prime}, s, s^{\prime}\right)$. One solution is $(\hat{a}, \hat{a}, 1,1)$ where $\hat{a}=(1-\lambda) /\left(r^{-1}-\lambda\right)$, which is a version of Holmström's (1999) Proposition 1. Now let's treat $s$ as a parameter to begin with. Investment as a function of $s$ is given by

$$
a(s)=\frac{r(1-\lambda)}{1-(r \lambda)^{2}}(r \lambda+s), \text { and } a^{\prime}(s)=\frac{r(1-\lambda)}{1-(r \lambda)^{2}}\left(r \lambda+s^{-1}\right) .
$$

Thus our problem is equivalent to looking for a fixed point in $s$ of the function $\psi(s)$ defined in (42).

Proof. Proposition 7: Since $U(c)=-\exp (-\gamma c)$, it follows from the definition in (42) of $\psi(\cdot)$ that $\psi(1)=1$. Let $\bar{s} \equiv 2 \frac{1-(r \lambda)^{2}}{r(1-\lambda)}-r \lambda$, since $c(\bar{s})=0$ and $c(1 / \bar{s}) \in(0,1)$, we have $\psi(\bar{s})<1$. By continuity of the mapping $\psi(\cdot)$, there will be a fixed point $s \in(1, \bar{s})$ if $\psi^{\prime}(1)>1$. Differentiating $\psi(\cdot)$, we obtain

$\psi^{\prime}(s)=\gamma\left(1-a(s)+\frac{1-a(1 / s)}{s^{2}}\right) \frac{r(1-\lambda)}{1-(r \lambda)^{2}} \exp \left(\gamma\left[a(s)-\frac{a(s)^{2}}{2}-\left(a(1 / s)-\frac{a(1 / s)^{2}}{2}\right)\right]\right)$,

and so

$$
\psi^{\prime}(1)>1 \Leftrightarrow 2 \gamma[1-a(1)] \frac{r(1-\lambda)}{1-(r \lambda)^{2}}>1 .
$$

This expression can be simplified as $a(1)=\hat{a}=(1-\lambda) /\left(r^{-1}-\lambda\right)$ so that

$$
\frac{r(1-\lambda)}{1-(r \lambda)^{2}}=\frac{\hat{a}}{1+r \lambda}=\frac{a(1)}{1+r \lambda}
$$

Reinserting this equality into the previous equation we get

$$
\psi^{\prime}(1)>1 \Leftrightarrow 2 \gamma[1-a(1)] a(1)>1+r \lambda,
$$


which implies in turn that

$$
\frac{\frac{1}{a(1)}-\gamma(1-a(1))}{\frac{r \lambda}{a(1)}-\gamma(1-a(1))} \in(-1,1) .
$$

This allows us to conclude that the rest point is stable since equation (40) with quadratic costs and CARA utility reads

$$
\varphi^{\prime}(a(1))=\left.\frac{d a_{t+1}}{d a_{t}}\right|_{a_{t}=a(1)}=\frac{\frac{1}{a(1)}-\gamma(1-a(1))}{\frac{1}{\frac{1-\lambda}{\lambda}+a(1)}-\gamma(1-a(1))}=\frac{\frac{1}{a(1)}-\gamma(1-a(1))}{\frac{r \lambda}{a(1)}-\gamma(1-a(1))},
$$

where the last equality follows from expression of the rest-point $a(1)=(1-\lambda) /\left(r^{-1}-\right.$ $\lambda)$.

\section{A.3 Asymmetric cycles.}

Proposition 8 derives conditions under which cycles of period 3 can be constructed using the partial equilibrium model with linear costs described in Subsection 3. It focuses on asymmetric cycles with protracted booms and sudden busts. The logic of the proof can be applied to study reverse cases and to show that cycles with protracted slumps and sudden booms can be sustained when $\kappa>(1-\lambda) /\left(r^{-1}-\lambda\right)$. Furthermore, it is cumbersome but relatively straightforward to extend the proof so as to construct cycles with more than 3 states.

Proposition 8 Consider the partial equilibrium model with CARA utility and linear costs, i.e., $g(a)=\kappa a$. Assume that $\kappa$ satisfies the compatible conditions

$$
\frac{1-\lambda}{r^{-1}-\lambda}>\kappa>\frac{r(1-\lambda)}{1-(r \lambda)^{3}}\left[\frac{r^{2} \lambda(1-\lambda+\lambda \kappa)}{\kappa}+(r \lambda)^{2}\right] .
$$

Then there exists a unique $\bar{a}_{\min }>0$ such that deterministic cycles of period three, where

$$
a_{t}^{*}=\left\{\begin{array}{l}
\bar{a} \text { when } t=\{0,3,6, \ldots\} \\
0 \text { when } t=\{1,4,7, \ldots\} \\
\tilde{a} \in(0, \bar{a}) \text { when } t=\{2,5,8, \ldots\},
\end{array}\right.
$$

are sustainable whenever $\bar{a} \geq \bar{a}_{\text {min }}$. 
Proof. Proposition 8: We propose a constructive proof. We study each action in turn and prove their incentive compatibility

1. $a_{t}^{*}=\bar{a}$ : Since we are focusing on cases where $\kappa<r(1-\lambda) /(1-r \lambda)$, we have

$$
\kappa \leq \frac{1-\lambda}{\lambda} \sum_{i=\{1,2, \ldots\}}^{\infty}(r \lambda)^{i}<\frac{1-\lambda}{\lambda} \sum_{i=\{1,2, \ldots\}}(r \lambda)^{i} S\left(\bar{a}-a_{t+i}^{*}\right) .
$$

The last inequality follows from the definition of $S(\cdot)$ in (36) because $\bar{a} \geq a_{t+i}^{*}$ for all $i$, and so $S\left(\bar{a}-a_{t+i}^{*}\right) \geq 1$, with strict inequality for some $i$. Since the last expression in (49) measures the discounted returns from effort at date $t$, the feasibility constraint binds and $a_{t}^{*}=\bar{a}$ is indeed incentive compatible.

2. $a_{t}^{*}=0$ : At the lower-bound of the feasibility set, costs must exceed returns so that

$$
\begin{aligned}
\kappa & \geq \frac{1-\lambda}{\lambda} \sum_{i=1,2, \ldots}^{\infty}(r \lambda)^{i} S\left(-a_{t+i}^{*}\right) \\
& =S(-\tilde{a}) r\left(1-\lambda+\lambda\left[\frac{1-\lambda}{\lambda} \sum_{i=\{2,3, \ldots\}}(r \lambda)^{i-1} S\left(\tilde{a}-a_{t+i}^{*}\right)\right]\right) \\
& =S(-\tilde{a}) r(1-\lambda+\lambda \kappa) .
\end{aligned}
$$

The last equality follows from the fact that the incentive constraint must hold with equality in the next period because $a_{t+1}^{*}=\tilde{a} \in(0, \bar{a})$. The condition is satisfied whenever

$$
\tilde{a} \geq \tilde{a}_{\min } \equiv \frac{-\log \left(\frac{\kappa}{r(1-\lambda+\lambda \kappa)}\right)}{\gamma(1-\kappa)}>0 .
$$

3. $a_{t}^{*}=\tilde{a} \in(0, \bar{a})$ : First, we assume that condition (50) holds as an equality and we show that there exists a unique value of $\bar{a}$ which renders $\tilde{a}_{m i n}$ incentive compat- 
ible. Since $\tilde{a}$ is interior, the incentive constraint has to hold exactly, i.e.,

$$
\begin{aligned}
\kappa & =\frac{1-\lambda}{\lambda} \sum_{i=1,2, \ldots}^{\infty}(r \lambda)^{i} S\left(\tilde{a}-a_{t+i}^{*}\right) \\
& =S(\tilde{a}-\bar{a}) r\left(1-\lambda+\lambda\left[\frac{1-\lambda}{\lambda} \sum_{i=\{2,3, \ldots\}}(r \lambda)^{i-1} S\left(\bar{a}-a_{t+i}^{*}\right)\right]\right) \\
& >S(\tilde{a}-\bar{a}) r(1-\lambda+\lambda \kappa)>S(\tilde{a}-\bar{a}) \kappa .
\end{aligned}
$$

The first inequality follows from step 1 above, while the second one holds true because $\kappa<r(1-\lambda) /(1-r \lambda)$. It shows that $S(\tilde{a}-\bar{a})$ has to be inferior to one, thus requiring that $\bar{a}$ be strictly larger than $\tilde{a}$. Keeping $\tilde{a}$ constant and differentiating (51) with respect to $\bar{a}$, we find that returns are strictly decreasing in $\bar{a}$. Furthermore, if

$$
\kappa>\frac{1-\lambda}{\lambda}\left[\frac{(r \lambda)^{2} r(1-\lambda+\lambda \kappa) / \kappa+(r \lambda)^{3}}{1-(r \lambda)^{3}}\right],
$$

there exists a unique value, which we denote $\bar{a}_{\min }$, such that $\tilde{a}=\tilde{a}_{\min }$ and (51) is satisfied. To verify that (52) is consistent with $r(1-\lambda) /(1-r \lambda)>\kappa$, notice that

$$
\frac{r(1-\lambda)}{1-r \lambda}>\frac{1-\lambda}{\lambda}\left[\frac{(r \lambda)^{2} r(1-\lambda+\lambda \kappa) / \kappa+(r \lambda)^{3}}{1-(r \lambda)^{3}}\right] \Leftrightarrow 1>r \lambda\left[\frac{r(1-\lambda+\lambda \kappa)}{\kappa}-1\right] .
$$

This inequality is equivalent to $\kappa>r^{2} \lambda(1-\lambda) /\left[1+r \lambda-(r \lambda)^{2}\right]$, which yields a lower-bound that is inferior to the term on the right-hand side of (48) as well as to $r(1-\lambda) /(1-r \lambda)$. Hence the two conditions in (48) are compatible since they define a non-empty interval. As with period-2 cycles, low levels of effort can never be incentive compatible when costs are too small.

We still have to prove that our cycles are sustainable when $\bar{a}>\bar{a}_{\min }$. Differentiating (51) with respect to both $\bar{a}$ and $\tilde{a}$, one finds that $\partial \tilde{a} / \partial \bar{a} \in(0,1)$. The derivative being positive, $\tilde{a}>\tilde{a}_{\min }$ if $\bar{a}>\bar{a}_{m i n}$, and the condition (50) for incentive compatibility of $a_{t}^{*}=0$ is satisfied. Furthermore, the derivative being smaller than one ensures that $\tilde{a}$ remains within the interior of the feasible set as $\bar{a}$ increases. 


\section{A.4 Stochastic cycles.}

Proposition 9 : Let the effort pair $(\underline{a}(s), \bar{a}(s))$ be defined as follows

$$
\begin{aligned}
& \underline{a}(s)=r(1-\lambda) \alpha\left[\frac{r \lambda \alpha+s+(1-\alpha)(1-r \lambda(1-\alpha)) / \alpha}{(1-r \lambda(1-\alpha))^{2}-(r \lambda \alpha)^{2}}\right] \\
& \bar{a}(s)=r(1-\lambda) \alpha\left[\frac{r \lambda \alpha+s^{-1}+(1-\alpha)(1-r \lambda(1-\alpha)) / \alpha}{(1-r \lambda(1-\alpha))^{2}-(r \lambda \alpha)^{2}}\right] .
\end{aligned}
$$

If the fixed point problem

$$
s=\frac{U^{\prime}(c(\bar{a}(s))-g(\bar{a}(s)))}{U^{\prime}(c(\underline{a}(s))-g(\underline{a}(s)))}
$$

admits a solution $s^{*} \neq 1$, stochastic cycles where effort switches with probability $\alpha \in$ $(0,1)$ from $\underline{a}(s)$ to $\bar{a}(s)$, or from $\bar{a}(s)$ to $\underline{a}(s)$, are sustainable

Proof. Proposition 9: Let $s=U^{\prime}(c(\bar{a})-g(\bar{a})) / U^{\prime}(c(\underline{a})-g(\underline{a}))$ denote the discount factor when effort switches from $\underline{a}$ to $\bar{a}$. Then the recursive incentive constraint (18) is satisfied when the effort pair $(\underline{a}, \bar{a})$ solves the following system of equations

$$
\begin{aligned}
& \underline{a}=r[(1-\alpha)(1-\lambda+\lambda \underline{a})+\alpha s(1-\lambda+\lambda \bar{a})], \\
& \bar{a}=r\left[(1-\alpha)(1-\lambda+\lambda \bar{a})+\alpha s^{-1}(1-\lambda+\lambda \underline{a})\right] .
\end{aligned}
$$

Combining the two equations above yields (53) and (54). As expected, when $\alpha=1$, we recover the expressions (45) and (46) in Lemma 2. 